\title{
Mean-squared error estimation in transformed Fay-Herriot models
}

\author{
Eric V. Slud \\ University of Maryland, College Park, USA \\ and Tapabrata Maiti \\ lowa State University, Ames, USA
}

[Received June 2004. Revised October 2005]

\begin{abstract}
Summary. The problem of accurately estimating the mean-squared error of small area estimators within a Fay-Herriot normal error model is studied theoretically in the common setting where the model is fitted to a logarithmically transformed response variable. For bias-corrected empirical best linear unbiased predictor small area point estimators, mean-squared error formulae and estimators are provided, with biases of smaller order than the reciprocal of the number of small areas. The performance of these mean-squared error estimators is illustrated by a simulation study and a real data example relating to the county level estimation of child poverty rates in the US Census Bureau's on-going 'Small area income and poverty estimation' project.
\end{abstract}

Keywords: Fay-Herriot model; Non-linearly transformed responses; Second-order correction; Small area estimation; Survey estimation

\section{Introduction}

Small area estimation is becoming increasingly important in survey applications, particularly in those fields of official statistics where legislative mandates require socioeconomic estimates within narrower jurisdictions than can accurately be described by direct estimates from national surveys. Because of the potentially large effect of decisions that are made by using survey results, it is important that estimates be reported together with their precisions, and the mean-squared error (MSE) is the measure of precision which has become standard in the small area field (Rao, 2003).

Especially where surveys measure variables such as population counts or amounts of money monitored for fractional changes, small area models involving these variables are often specified in terms of logarithmic or other transformations applied to sampled data. After the transformation of sampled responses, applying the inverse transformation to the small area estimates of transformed responses can yield good estimates of the small area responses after bias correction. Transformations will sometimes be chosen so that confidence intervals for small area parameters will be approximately symmetric on the transformed scale but not on the original scale. Nevertheless, MSEs and coefficients of variation are still a standard way of reporting the precision of survey estimates and may be much more interpretable on the meaningful rather than the transformed scale of measurement.

There has until now been no extension of the very useful theories of Prasad and Rao (1990) and Datta and Lahiri (2001) on 'second-order correct' estimation of MSEs of Fay-Herriot small

Address for correspondence: Eric V. Slud, Mathematics Department, University of Maryland at College Park, College Park, MD 20742, USA.

E-mail: evs@math.umd.edu

Royal Statistical Society 
area estimators to the case of non-linearly transformed responses. Jiang et al. (2002) proposed a jackknife-based bias correction for valid MSE estimates which leads to an estimate of the MSE of the best predictor, although generally not of the best linear unbiased predictor. Maiti (2004) applied a jackknife method of MSE estimation in an empirical Bayes framework, using lognormal models in which moments could be easily calculated. Rao (2003), page 133, indicated the non-triviality of extending to the transformed response case the approach of Prasad-Rao and Datta-Lahiri to MSE estimation via Taylor linearization. Extended second-order MSE formulae and estimators in this setting based on linearization comprise the main contribution of the present paper.

The paper is organized as follows. The Fay and Herriot (1979) model as applied to transformed data is presented in Section 2 along with the bias-corrected small area estimation formulae. Section 3 develops the theoretical expressions for MSE of the small area point estimators, asymptotically for large numbers $m$ of small areas. The derivations follow a novel approach even in the case of untransformed Fay-Herriot data, but they show their real usefulness in the extensions to non-linearly, particularly logarithmically, transformed data. Section 4 provides estimators of the MSE for small area estimates (SAEs), which are unbiased up to order $\mathcal{O}(1 / m)$. A simulation study in Section 5 strongly supports the theoretically predicted behaviours of our MSE estimators even in studies with as few as 100 small areas. Next, a data analytic application of the MSE estimates in a real data set is given in Section 6. Finally, we draw together our conclusions and recommendations in Section 7.

\section{Fay-Herriot models for small area estimation}

The mixed effect linear models that are studied here for small area applications are all based on the Fay and Herriot (1979) model form. For each area indexed by $i=1, \ldots, m$, assume that sample sizes $n_{i}$ and $p$-dimensional vectors $x_{i}$ of predictor variables are known, and that response variables satisfying

$$
y_{i}=x_{i}^{\operatorname{tr}} \beta+u_{i}+e_{i}, \quad u_{i} \sim \mathcal{N}\left(0, \sigma^{2}\right), \quad e_{i} \sim \mathcal{N}\left(0, s_{i}\right),
$$

are observed (whenever $n_{i}>0$ ), where $\beta \in \mathbf{R}^{p}$ is a vector of unknown fixed effect coefficients and $u_{i}$ and $e_{i}$ are respectively area random effects and sampling errors, independent of each other within and across areas. The variances $s_{i}$ are assumed to be known functions of $n_{i}$ except possibly for a constant $v_{e}$ of proportionality: the usual form is $s_{i} \equiv v_{e} / n_{i}$. Ordinarily, $\sigma^{2}$ is unknown and estimated, whereas $v_{e}$ is known. In some applications, such as that of Section 6 , it also makes sense to treat $\sigma^{2}$ as known (estimated from an auxiliary model fitted to the most recent decennial census data; see Citro and Kalton (2000), appendix A) and $v_{e}$ as unknown, but we do not give explicit formulae for that case here.

$S A E s$ based on such Fay-Herriot models are statistics that are designed to estimate with small MSE the parameters

$$
\vartheta_{i}=x_{i}^{\mathrm{tr}} \beta+u_{i}, \quad i=1, \ldots, m .
$$

In log-transformed Fay-Herriot models, $y_{i}$ is the observed log-response for the $i$ th area, with the interpretable small area parameter defined by exponentiating:

$$
\vartheta_{i}^{*}=\exp \left(\vartheta_{i}\right) \equiv \exp \left(x_{i}^{\operatorname{tr}} \beta+u_{i}\right)
$$

\subsection{Small area estimation formulae}

In the Fay-Herriot model, the estimators that we consider for $\vartheta_{j}$ based on the data $\left\{y_{i}, n_{i}: n_{i}>\right.$ $0,1 \leqslant i \leqslant m\}$ above are the estimated best linear unbiased predictor estimators (Rao, 2003) 


$$
\hat{\vartheta}_{j}=x_{j}^{\operatorname{tr}} \hat{\beta}+\hat{\gamma}_{j}\left(y_{j}-x_{j}^{\operatorname{tr}} \hat{\beta}\right)
$$

where $\left(\hat{\beta}, \hat{\sigma}^{2}\right)$ or $\left(\hat{\beta}, \hat{v}_{e}\right)$ are the maximum likelihood estimators in model (1) and

$$
\hat{\gamma}_{j}=\frac{\hat{\sigma}^{2}}{\hat{\sigma}^{2}+v_{e} / n_{j}} .
$$

We follow the convention that $\hat{\gamma}_{j} \equiv 0$ (so that $\hat{\vartheta}_{j}=x_{j}^{\mathrm{tr}} \hat{\beta}$ ) when $n_{j}=0$. In addition, we define for future reference $s_{i}=v_{e} / n_{i}, \tau_{i}=\sigma^{2}+s_{i}, \gamma_{i}=\sigma^{2} / \tau_{i}, \eta_{i}=x_{i}^{\operatorname{tr}} \beta$ and $\hat{\eta}_{i}=x_{i}^{\operatorname{tr}} \hat{\beta}$.

We present results both for the usual Fay-Herriot model, with errors evaluated directly between $\vartheta_{j}, \hat{\vartheta}_{j}$, and also with errors measured between

$$
\vartheta_{j}^{*}=h\left(\vartheta_{j}\right)
$$

and a direct estimator of this transformed parameter, where $h(x)$ is a known smooth monotone function $\left(\exp (x)\right.$ for most of the current paper). In the transformed case, the parameters $\vartheta_{j}^{*}$ are estimated by the approximately bias-corrected formula

$$
\hat{\vartheta}_{j}^{*}=\hat{\rho} h\left(\hat{\vartheta}_{j}\right), \quad \hat{\rho}=\hat{E}\left\{h\left(\vartheta_{j}\right)\right\} / \hat{E}\left\{h\left(\hat{\vartheta}_{j}\right)\right\},
$$

where the notation $\hat{E}(\cdot)$ refers to an estimator that is obtained by substituting $\left(\hat{\beta}, \hat{\sigma}^{2}\right)$ for $\left(\beta, \sigma^{2}\right)$ within an asymptotically correct expression for the specified expectation. In the most interesting case, with $h(x)=\exp (x)$, the formula (4) is

$$
\hat{\vartheta}_{j}^{*}=\exp \left\{x_{j}^{\operatorname{tr}} \hat{\beta}+\hat{\gamma}_{j}\left(y_{j}-x_{j}^{\operatorname{tr}} \hat{\beta}\right)+\frac{1}{2} \hat{\sigma}^{2}\left(1-\hat{\gamma}_{j}\right)\right\},
$$

which ignores the variability of the parameter estimators but corrects bias due to the area random effect. With general $h$, the analogous formula is

$$
\hat{\vartheta}_{j}^{*}=\left.h\left(\hat{\vartheta}_{j}\right) \frac{E\left\{h\left(\eta_{j}+u_{j}\right)\right\}}{E\left[h\left\{\eta_{j}+\gamma_{j}\left(u_{j}+e_{j}\right)\right\}\right]}\right|_{\left(\beta, \sigma^{2}\right)=\left(\hat{\beta}, \hat{\sigma}^{2}\right)} .
$$

\section{Mean-squared error formulae}

In this section, we provide a large sample approximation to the MSE in model (1) which is closely related to standard expressions for maximum likelihood estimates (MLEs) in terms of sums of independent terms. It is assumed throughout this section that the response variables $y_{i}, i=1, \ldots, m$, satisfy model (1), and also that

(a) the random vectors $x_{i}$ and sample sizes $n_{i}$ are either uniformly bounded or are realizations of independent identically distributed variates with finite fourth moments,

(b) whether random or not, the vectors $x_{i} \in \mathbf{R}^{p}$ are such that (with probability approaching 1) as $m \rightarrow \infty, \sum_{i=1}^{m} x_{i}^{\otimes 2}$ is a positive definite matrix, where, for a column vector $\mathbf{v}$, we denote $\mathbf{v}^{\otimes 2}=\mathbf{v v}^{\text {tr }}$ and

(c) the following large sample (in-probability) limits exist as $m \rightarrow \infty$, with error terms $O_{P}\left(m^{-1 / 2}\right)$ :

$$
\begin{gathered}
\bar{I}^{(1)}=\lim _{m}\left(m^{-1} \sum_{i=1}^{m} \frac{x_{i}^{\otimes 2}}{\sigma^{2}+s_{i}}\right), \\
\bar{I}^{(2)}=\lim _{m}\left\{m^{-1} \sum_{i=1}^{m} \frac{1}{2\left(\sigma^{2}+s_{i}\right)^{2}}\right\} .
\end{gathered}
$$

The log-likelihood for the data is given by 


$$
\operatorname{loglik}=-\frac{1}{2} \sum_{i=1}^{m} \log \left(\sigma^{2}+s_{i}\right)-\sum_{i=1}^{m} \frac{\left(y_{i}-x_{i}^{\mathrm{tr}} \beta\right)^{2}}{2\left(\sigma^{2}+s_{i}\right)}
$$

and is partially maximized (in $\beta$, for fixed $\sigma^{2}$ ) when

$$
\beta=\hat{\beta}\left(\sigma^{2}\right) \equiv\left(\sum_{i=1}^{m} \frac{x_{i}^{\otimes 2}}{\sigma^{2}+s_{i}}\right)^{-1} \sum_{i=1}^{m} \frac{x_{i} y_{i}}{\sigma^{2}+s_{i}} .
$$

The log-likelihood with $\hat{\beta}\left(\sigma^{2}\right)$ substituted for $\beta$ is called the profile log-likelihood, and it is maximized over $\sigma^{2}$ to generate the estimator $\hat{\sigma}^{2}$.

The large sample information matrix $I^{(F)}$ for $\left(\beta, \sigma^{2}\right)$ based on the data is easily calculated to have the block-decomposed form

$$
\mathcal{I}=\left(\begin{array}{cc}
\Sigma_{\hat{\beta}}^{-1} & \mathbf{0} \\
\mathbf{0}^{\mathrm{tr}} & \Sigma_{\hat{\sigma}^{2}}^{-1}
\end{array}\right), \quad \Sigma_{\hat{\beta}}^{-1}=\sum_{i=1}^{m} \frac{x_{i}^{\otimes 2}}{\sigma^{2}+s_{i}}, \quad \Sigma_{\hat{\sigma}^{2}}^{-1}=\sum_{i=1}^{m} \frac{1}{2\left(\sigma^{2}+s_{i}\right)^{2}}
$$

and, by assumption (c), the limiting per-observation information $m^{-1} \mathcal{I}$ exists and is block diagonal with blocks $\bar{I}^{(1)}$ and $\bar{I}^{(2)}$. Then the score statistic vector is easily calculated to be

$$
\nabla_{\beta, \sigma^{2}} \log \mathrm{lik}=\sum_{i=1}^{m} \frac{1}{2\left(\sigma^{2}+s_{i}\right)}\left(\begin{array}{c}
2 x_{i}\left(y_{i}-x_{i}^{\mathrm{tr}} \beta\right) \\
-1+\left(y_{i}-x_{i}^{\mathrm{tr}} \beta\right)^{2} /\left(\sigma^{2}+s_{i}\right)
\end{array}\right)
$$

and a standard general result — based on simple Taylor series expansion for locally consistent maximum likelihood estimates - gives

$$
\sqrt{ } m\left(\begin{array}{c}
\hat{\beta}-\beta \\
\hat{\sigma}^{2}-\sigma^{2}
\end{array}\right)=\sum_{i=1}^{m} \frac{\sqrt{ } m}{\tau_{i}}\left(\begin{array}{c}
\Sigma_{\hat{\beta}} x_{i}\left(u_{i}+e_{i}\right) \\
\Sigma_{\hat{\sigma}^{2}} \frac{\left(u_{i}+e_{i}\right)^{2}-\tau_{i}}{2 \tau_{i}}
\end{array}\right)+o_{P}(1)
$$

with the $o_{P}(1)$ remainder holding in probability under model (1) with actual parameters $\left(\beta, \sigma^{2}\right)$. However, we require slightly greater precision than is provided by equation (7). More careful Taylor series expansion about $\left(\beta, \sigma^{2}\right)$ of the likelihood equation (setting the gradient of the log-likelihood equal to 0 at the MLE), using the form of the log-likelihood under model (1), shows that the error in equation (7) is actually $\mathcal{O}_{P}\left(m^{-1 / 2}\right)$. We justify this in the next result, the proof of which can be found in Appendix A.

Lemma 1. In the normal errors Fay-Herriot model (1),

$$
\begin{aligned}
\hat{\beta}-\beta-\Sigma_{\hat{\beta}} \sum_{i=1}^{m} \frac{x_{i}}{\tau_{i}}\left(u_{i}+e_{i}\right)= & -\Sigma_{\hat{\beta}} \sum_{i=1}^{m} \frac{x_{i}}{\tau_{i}^{2}}\left(u_{i}+e_{i}\right) \Sigma_{\hat{\sigma}^{2}} \sum_{i=1}^{m} \frac{\left(u_{i}+e_{i}\right)^{2}-\tau_{i}}{2 \tau_{i}^{2}} \\
& +\Sigma_{\hat{\beta}} \sum_{i=1}^{m} \frac{x_{i}^{\otimes 2}}{\tau_{i}^{2}} \Sigma_{\hat{\beta}} \sum_{i=1}^{m} \frac{x_{i}}{\tau_{i}}\left(u_{i}+e_{i}\right) \Sigma_{\hat{\sigma}^{2}} \sum_{i=1}^{m} \frac{\left(u_{i}+e_{i}\right)^{2}-\tau_{i}}{2 \tau_{i}^{2}}+o_{P}\left(\frac{1}{m}\right)
\end{aligned}
$$

and

$$
\begin{aligned}
\Sigma_{\hat{\sigma}^{2}}^{-1}\left\{\hat{\sigma}^{2}-\sigma^{2}-\Sigma_{\hat{\sigma}^{2}} \sum_{i=1}^{m} \frac{\left(u_{i}+e_{i}\right)^{2}-\tau_{i}}{2 \tau_{i}^{2}}\right\} \\
=\sum_{i=1}^{m}\left[\frac{-\left(u_{i}+e_{i}\right) x_{i}^{\mathrm{tr}}}{\tau_{i}^{2}}(\hat{\beta}-\beta)+\frac{\left\{x_{i}^{\mathrm{tr}}(\hat{\beta}-\beta)\right\}^{2}}{2 \tau_{i}^{2}}+\frac{\left(\hat{\sigma}^{2}-\sigma^{2}\right)^{2}}{\tau_{i}^{3}}\right]-\Sigma_{\hat{\sigma}^{2}} \sum_{i=1}^{m} \frac{\left(u_{i}+e_{i}\right)^{2}-\tau_{i}}{2 \tau_{i}^{2}} \\
\quad \times \sum_{i=1}^{m} \frac{\left(u_{i}+e_{i}\right)^{2}-\tau_{i}}{\tau_{i}^{3}}+o_{P}(1)
\end{aligned}
$$


Corollary 1. The bias of the estimators $\hat{\beta}$ and $\hat{\sigma}^{2}$, up to order $1 / m$ terms, is given by the formulae

$$
\begin{gathered}
E(\hat{\beta}-\beta)=o\left(\frac{1}{m}\right), \\
E\left(\hat{\sigma}^{2}-\sigma^{2}\right)=-\Sigma_{\hat{\sigma}^{2}} \sum_{i=1}^{m} \frac{x_{i}^{\operatorname{tr}} \Sigma_{\hat{\beta}} x_{i}}{2 \tau_{i}^{2}}+o\left(\frac{1}{m}\right) .
\end{gathered}
$$

Corollary 1 follows immediately from equations (8) and (9), after checking the uniform integrability of the remainders for $m(\hat{\beta}-\beta)$ and $m\left(\hat{\sigma}^{2}-\sigma^{2}\right)$ in the proof of lemma 1 . The next lemma leads to an effective grouping of terms in the expansion of $\hat{\vartheta}_{j}-\vartheta_{j}$ and is proved in Appendix A.

Lemma 2. Define for each $i=1, \ldots, m$,

$$
\alpha_{i}=\gamma_{i} e_{i}-\left(1-\gamma_{i}\right) u_{i}
$$

Then $\alpha_{j}$ is conditionally independent of $\left\{u_{i}+e_{i}\right\}_{i=1}^{m}$ given $\left\{\left(x_{i}, n_{i}\right)\right\}_{i=1}^{m}$, whereas the large sample consistent root $\left(\hat{\beta}, \hat{\sigma}^{2}\right)$ of likelihood equations (25)-(26) depends on variables $\left\{\left(u_{i}, e_{i}\right)\right\}_{i=1}^{m}$ through $\left\{u_{i}+e_{i}\right\}_{i=1}^{m}$ alone.

From equations (7)-(9), we obtain the large sample approximate conditional distribution for the MLEs given $\left(u_{j}, e_{j}\right)$ for each fixed $j=1, \ldots, m$.

Theorem 1. For fixed $j \in\{1, \ldots, m\}$, define

$$
\begin{aligned}
\mathbf{Z}_{j} & \equiv\left(\begin{array}{c}
Z_{j}^{(1)} \\
Z_{j}^{(2)}
\end{array}\right) \\
& =\sum_{i: i \neq j} \frac{1}{\sigma^{2}+s_{i}}\left(\begin{array}{c}
\sum_{\hat{\beta}}^{1 / 2} x_{i}\left(u_{i}+e_{i}\right) \\
\frac{1}{2} \Sigma_{\hat{\sigma}^{2}}^{1 / 2}\left\{\frac{\left(u_{i}+e_{i}\right)^{2}}{\sigma^{2}+s_{i}}-1\right\}
\end{array}\right) .
\end{aligned}
$$

Then, for each $j$, asymptotically for large $m$ the random variables $Z_{j}^{(1)} \sim \mathcal{N}\left(\mathbf{0}, I d_{p}\right)$ and $Z_{j}^{(2)} \sim$ $\mathcal{N}(0,1)$ are independent of each other and of $\left(u_{j}, e_{j}\right)$ conditionally given $\left\{x_{i}, n_{i}: i=1, \ldots, m\right\}$. Moreover, for fixed $j$, recalling the notation $\tau_{j}=\sigma^{2}+s_{j}$,

$$
\left(\begin{array}{c}
\hat{\beta}-\beta-\Sigma_{\hat{\beta}}^{1 / 2} Z_{j}^{(1)}-\Sigma_{\hat{\beta}} x_{j}\left(u_{j}+e_{j}\right) / \tau_{j} \\
\hat{\sigma}^{2}-\sigma^{2}-\Sigma_{\hat{\sigma}^{2}}^{1 / 2} Z_{j}^{(2)}-\Sigma_{\hat{\sigma}^{2}}\left\{\left(u_{j}+e_{j}\right)^{2}-\tau_{j}\right\} / 2 \tau_{j}^{2}
\end{array}\right)=\mathcal{O}_{P}\left(\frac{1}{m}\right)
$$

and the right-hand side of equation (13) is equal to the vector concatenating the right-hand side of equation (8) with the right-hand side of equation (9) multiplied by $\Sigma_{\hat{\sigma}^{2}}$.

Proof. We separate out the terms with index $j$ within equation (7) from the others and recognize that the error arising on the right-hand side of equation (13) from replacing $\Sigma_{\hat{\beta}}$ and $\Sigma_{\hat{\sigma}^{2}}$ with (inverses of) the same sums omitting $i=j$ is of smaller order in probability than $m^{-1}$. Beyond that, the assertion follows immediately from the proof of lemma 1.

By means of theorem 1, we expand $\hat{\vartheta}_{j}$ into terms involving $u_{j}+e_{j}$, terms involving randomeffect variables with indices other than $j$, and remainders. We retain all the terms that are needed so that the remainder in $\hat{\vartheta}_{j}$ is of order $1 / m$ in probability and in expectation ( $L^{1}$-norm). 
Theorem 2. Under the hypotheses of this section, and the notation of theorem 1, as $m$ becomes large,

$$
\begin{aligned}
\hat{\vartheta}_{j}= & \vartheta_{j}+\alpha_{j}+\left(1-\gamma_{j}\right) x_{j}^{\operatorname{tr}} \Sigma_{\hat{\beta}}^{1 / 2} Z_{j}^{(1)}+\frac{1-\gamma_{j}}{\tau_{j}}\left(u_{j}+e_{j}\right) \Sigma_{\hat{\sigma}^{2}}^{1 / 2} Z_{j}^{(2)}+\frac{1-\gamma_{j}}{\tau_{j}}\left(u_{j}+e_{j}\right) \\
& \times\left[x_{j}^{\operatorname{tr}} \Sigma_{\hat{\beta}} x_{j}+\frac{1}{2 \tau_{j}} \Sigma_{\hat{\sigma}^{2}}\left\{\frac{\left(u_{j}+e_{j}\right)^{2}}{\tau_{j}}-1\right\}\right]+\left(1-\gamma_{j}\right) x_{j}^{\operatorname{tr}} \Sigma_{\hat{\beta}}\left\{-\sum_{i=1}^{m} \frac{x_{i}}{\tau_{i}^{2}}\left(u_{i}+e_{i}\right) \Sigma_{\hat{\sigma}^{2}}\right. \\
& \left.\times \sum_{i=1}^{m} \frac{\left(u_{i}+e_{i}\right)^{2}-\tau_{i}}{2 \tau_{i}^{2}}+\sum_{i=1}^{m} \frac{x_{i}^{\otimes 2}}{\tau_{i}^{2}} \Sigma_{\hat{\beta}} \sum_{i=1}^{m} \frac{x_{i}}{\tau_{i}}\left(u_{i}+e_{i}\right) \Sigma_{\hat{\sigma}^{2}} \sum_{i=1}^{m} \frac{\left(u_{i}+e_{i}\right)^{2}-\tau_{i}}{2 \tau_{i}^{2}}\right\} \\
& +\frac{\left(u_{j}+e_{j}\right) s_{j}}{\tau_{j}^{2}} \Sigma_{\hat{\sigma}^{2}} \sum_{i=1}^{m}\left\{\frac{-\left(u_{i}+e_{i}\right) x_{i}^{\mathrm{tr}}}{\tau_{i}^{2}} \Sigma_{\hat{\beta}}^{1 / 2} Z_{j}^{(1)}+\frac{x_{i}^{\mathrm{tr}} \Sigma_{\hat{\beta}} x_{i}}{2 \tau_{i}^{2}}\left(Z_{j}^{(1)}\right)^{2}+\frac{\Sigma_{\hat{\sigma}^{2}}\left(Z_{j}^{(2)}\right)^{2}}{\tau_{i}^{3}}\right\} \\
& -\frac{\left(u_{j}+e_{j}\right) s_{j}}{\tau_{j}^{2}} \Sigma_{\hat{\sigma}^{2}}^{2} \sum_{i=1}^{m} \frac{\left(u_{i}+e_{i}\right)^{2}-\tau_{i}}{2 \tau_{i}^{2}} \sum_{i=1}^{m} \frac{\left(u_{i}+e_{i}\right)^{2}-\tau_{i}}{\tau_{i}^{3}} \\
& -\frac{s_{j} x_{j}^{\mathrm{tr}}}{\tau_{j}^{2}} \Sigma_{\hat{\beta}}^{1 / 2} Z_{j}^{(1)} \Sigma_{\hat{\sigma}^{2}}^{1 / 2} Z_{j}^{(2)}-\frac{s_{j}\left(u_{j}+e_{j}\right)}{\tau_{j}^{3}} \Sigma_{\hat{\sigma}^{2}}\left(Z_{j}^{(2)}\right)^{2}+o o_{P}\left(\frac{1}{m}\right)
\end{aligned}
$$

and in the $\mathcal{O}_{P}(1 / m)$ terms of equation (14) all summations over $i$ can be changed to run only over $i: i \neq j$.

Using theorem 2, we expand $\mathrm{MSE}_{j} \equiv E\left(\hat{\vartheta}_{j}-\vartheta_{j}\right)^{2}$ and $\mathrm{MSE}_{j}^{*} \equiv E\left(\hat{\vartheta}_{j}^{*}-\vartheta_{j}^{*}\right)^{2}$ up to terms of order $1 / m$, with a view to developing 'second-order-corrected' estimators for MSE ${ }_{j}^{*}$. This expansion is new in the case of non-linearly transformed small area parameters $\vartheta_{j}^{*}=h\left(\vartheta_{j}\right)$, but by now well known in the untransformed case (Rao, 2003).

\subsection{Simplifications with untransformed data}

We begin by accounting for the extreme simplifications arising in the known theoretical expressions for $\mathrm{MSE}_{j}=E\left(\hat{\vartheta}_{j}-\vartheta_{j}\right)^{2}$ by comparison with the expression that is derived below in the non-linearly transformed case. The conditional independence of $\alpha_{j}$ from $\hat{\vartheta}_{j}-\vartheta_{j}-\alpha_{j}$ given $\left\{\left(x_{i}, n_{i}\right)\right\}_{i=1}^{m}$, which is obtained in lemma 2, allows most of the cross-terms in the MSE to disappear.

Lemma 3. Under model (1) and assumptions (a)-(c),

$$
\begin{aligned}
\mathrm{MSE} & =E\left(\hat{\vartheta}_{j}-\vartheta_{j}\right)^{2} \\
& =g_{1 j}+g_{2 j}+g_{3 j}+O\left(m^{-3 / 2}\right)
\end{aligned}
$$

where

$$
\begin{gathered}
g_{1 j}=E\left(\alpha_{j}^{2}\right)=s_{j} \gamma_{j}, \\
g_{2 j}=\left(1-\gamma_{j}\right)^{2} x_{j}^{\mathrm{tr}} \Sigma_{\hat{\beta}} x_{j}, \\
g_{3 j}=\frac{\left(1-\gamma_{j}\right)^{2}}{\tau_{j}} \Sigma_{\hat{\sigma}^{2}} .
\end{gathered}
$$

Proof. Fix and condition on $\left\{\left(n_{i}, x_{i}\right)\right\}_{i=1}^{m}$ : by lemma 2 and equation (14), the mean 0 variable $\alpha_{j}$ is independent of 


$$
\hat{\vartheta}_{j}-\vartheta_{j}-\alpha_{j}=\left(1-\gamma_{j}\right) x_{j}^{\operatorname{tr}} \Sigma_{\hat{\beta}}^{1 / 2} Z_{j}^{(1)}+\frac{1-\gamma_{j}}{\tau_{j}}\left(u_{j}+e_{j}\right) \Sigma_{\hat{\sigma}^{2}}^{1 / 2} Z_{j}^{(2)}+o_{P}\left(m^{-1 / 2}\right)
$$

in which the final remainder term is also easily seen to be $\mathcal{O}_{P}(1 / m)$. It follows immediately that $\mathrm{MSE}_{j}=E\left(\alpha_{j}^{2}\right)+E\left(\hat{\vartheta}_{j}-\vartheta_{j}-\alpha_{j}\right)^{2}$, and the terms

$$
\left(1-\gamma_{j}\right) x_{j}^{\operatorname{tr}} \Sigma_{\hat{\beta}}^{1 / 2} Z_{j}^{(1)}
$$

and

$$
\left(1-\gamma_{j}\right) \frac{u_{j}+e_{j}}{\tau_{j}} \Sigma_{\hat{\sigma}^{2}}^{1 / 2} Z_{j}^{(2)}
$$

have respective second moments $g_{2 j}$ and $g_{3 j}$ and expected product $o(1 / m)=\mathcal{O}\left(m^{-3 / 2}\right)$. The proof is complete.

These simplifications in theoretical MSE do not persist in the situation of interest in this paper, where estimator (4) is used to estimate the transformed small area parameter $\vartheta_{j}^{*}=h\left(\vartheta_{j}\right)$.

\subsection{Exponential transformation}

The formula for MSE in the log-transformed model cannot be obtained simply from a delta method adjustment using the formula in the untransformed (linear) case. We begin by calculating the top order MSE expression (which is analogous to $g_{1}$ alone from the linear case). These calculations, unlike those in the linear case, specifically use the log-normal moment formulae: for $Z \sim \mathcal{N}(0,1), k \geqslant 0$,

$$
E\left\{(a Z)^{k} \exp (a b Z)\right\}=\left.\frac{\mathrm{d}^{k}}{\mathrm{~d} t^{k}} \exp \left(\frac{a^{2} t^{2}}{2}\right)\right|_{t=b} .
$$

First we calculate, with ' $\approx$ ' denoting equality up to $o(1)$ remainder,

$$
\begin{aligned}
\operatorname{MSE}_{j}^{*} \equiv & E\left(\exp \left[\left(1-\hat{\gamma}_{j}\right) \frac{\hat{\sigma}^{2}}{2}+\hat{\eta}_{j}+\hat{\gamma}_{j}\left\{u_{j}+e_{j}-x_{j}^{\operatorname{tr}}(\hat{\beta}-\beta)\right\}\right]-\exp \left(\eta_{j}+u_{j}\right)\right)^{2} \\
= & E\left[\exp \left\{\eta_{j}+\left(u_{j}+e_{j}\right) \gamma_{j}+\frac{1}{2} \sigma^{2}\left(1-\gamma_{j}\right)\right\}-\exp \left(\eta_{j}+u_{j}\right)\right]^{2}+o(1) \\
\approx & \exp \left(2 \eta_{j}\right)\left[\exp \left\{\left(1-\gamma_{j}\right) \sigma^{2}+2 \gamma_{j} \sigma^{2}\right\}+\exp \left(2 \sigma^{2}\right)\right. \\
& \left.-2 \exp \left\{\frac{\sigma^{2}}{2}\left(1-\gamma_{j}\right)+\frac{s_{j}}{2} \gamma_{j}^{2}+\frac{\sigma^{2}}{2}\left(1+\gamma_{j}\right)^{2}\right\}\right] \\
= & \exp \left\{2\left(\eta_{j}+\sigma^{2}\right)\right\}\left[\exp \left\{-\left(1-\gamma_{j}\right) \sigma^{2}\right\}+1-2 \exp \left(-\frac{s_{j} \sigma^{2}}{\tau_{j}}\right)\right] \\
= & \exp \left\{2\left(\eta_{j}+\sigma^{2}\right)\right\}\left\{1-\exp \left(-\gamma_{j} s_{j}\right)\right\} .
\end{aligned}
$$

This preliminary result is the top order or $\mathcal{O}(1)$ term in the $\mathrm{MSE}_{j}^{*}$ expression on the exponentiated scale, defined in formula (17):

$$
\mathrm{MSE}_{j}^{*} \approx G_{1 j} \equiv \exp \left\{2\left(\eta_{j}+\sigma^{2}\right)\right\}\left\{1-\exp \left(-\gamma_{j} s_{j}\right)\right\}
$$

which can be estimated within $o_{P}(1)$ by

$$
\hat{G}_{1 j}=\exp \left\{2\left(\hat{\eta}_{j}+\hat{\sigma}^{2}\right)\right\}\left\{1-\exp \left(-\hat{\gamma}_{j} s_{j}\right)\right\} .
$$


As a corollary of the previous discussion, this estimator is unbiased up to remainder $\mathcal{O}_{P}(1 / m)$. The remainder is analysed in expression (22) below.

The full expression for MSE (17) in the exponential transformation case can now be calculated, as we do up to $\mathcal{O}_{P}(1 / \mathrm{m})$ remainders. We record for later reference the simplified formulae from equation (16) for several terms which arise in $\mathrm{MSE}_{j}^{*}$.

Lemma 4.

(a) For $k \geqslant 0, j=1, \ldots, m$,

$$
E\left\{\left(u_{j}+e_{j}\right)^{k}\left(\exp \left[\gamma_{j}\left\{s_{j}+2\left(u_{j}+e_{j}\right)\right\}\right]-\exp \left\{\gamma_{j}\left(\frac{s_{j}}{2}+u_{j}+e_{j}\right)+u_{j}\right\}\right)\right\}=0 .
$$

(b) For $0 \leqslant k \leqslant 2$,

$$
\begin{aligned}
\mu_{j k} & \equiv E\left(\left(u_{j}+e_{j}\right)^{k} \exp \left[\gamma_{j}\left\{s_{j}+2\left(u_{j}+e_{j}\right)\right\}\right]\right) \\
& =\exp \left\{\sigma^{2}\left(1+\gamma_{j}\right)\right\} \times \begin{cases}1 & \text { for } k=0, \\
2 \sigma^{2} & \text { for } k=1, \\
4 \sigma^{4}+\tau_{j} & \text { for } k=2 .\end{cases}
\end{aligned}
$$

Now all terms in equation (14) up to order $m^{-1}$ are substituted for $\hat{\vartheta}_{j}^{*}$ within $\mathrm{MSE}_{j}^{*}$. As is shown in detail in Appendix A, this leads to the following simplified expression for MSE $\mathrm{M}_{j}^{*}$.

Theorem 3. Under the same assumptions as in theorem 2,

$$
\begin{aligned}
\operatorname{MSE}_{j}^{*}= & \exp \left\{2\left(\eta_{j}+\sigma^{2}\right)\right\}\left\{1-\exp \left(-\gamma_{j} s_{j}\right)\right\}+\frac{s_{j}^{2}}{\tau_{j}^{2}} \exp \left\{2 \eta_{j}+\sigma^{2}\left(1+\gamma_{j}\right)\right\} x_{j}^{\mathrm{tr}} \Sigma_{\hat{\beta}} x_{j} \\
& +\Sigma_{\hat{\sigma}^{2}} \frac{s_{j}^{2}}{\tau_{j}^{2}} \exp \left\{2 \eta_{j}+\sigma^{2}\left(1+\gamma_{j}\right)\right\}\left\{\frac{1}{4}\left(1+3 \gamma_{j}\right)^{2}+\frac{1}{\tau_{j}}\right\}+\mathcal{O}\left(m^{-3 / 2}\right) .
\end{aligned}
$$

Remark 1. The result of theorem 3 incorporates numerous simplifications, including especially those due to lemma 4 , arising when the transformation $h$ is exponential. For fully general smooth and monotone non-linear transformations $h$, theorem 3 has a less simple analogue. The general formulae depend, as before, on the expansion of $\hat{\vartheta}_{j}^{*}-\vartheta_{j}^{*}$, which involves Taylor series expansions around $\left(\beta, \sigma^{2}\right)$ of the expectation terms in equation (6), together with the expansion equation (14) of $\hat{\vartheta}_{j}$ in theorem 2 . The formulae, with details of proof and associated second-order-corrected estimators, are worked out in Slud and Maiti (2005).

Remark 2. Our results rely heavily on the assumed normal distribution of the area random effects $u_{i}$ in the Fay-Herriot model (1). In the untransformed case, it is known from Lahiri and Rao (1995) that second-order corrected MSE estimators based on method-of-moments empirical best linear unbiased predictor SAEs are robust to non-normality in $u_{i}$, but analogous results do not exist for SAEs that are based on MLE empirical best linear unbiased predictors as in Datta and Lahiri (2001).

\section{Mean-squared error estimation}

\subsection{Linear (untransformed) case}

We begin in the untransformed case, considering estimators with MLEs substituted for parameters in expression (15). The naïve empirical best linear unbiased predictor estimator would be $\hat{g}_{1 j}+\hat{g}_{2 j}+\hat{g}_{3 j}$, where (in terms of $\hat{\tau}_{i} \equiv \hat{\sigma}^{2}+s_{i}$ ) 


$$
\hat{g}_{1 j}=\frac{s_{j} \hat{\sigma}^{2}}{\hat{\tau}_{j}}, \quad \hat{g}_{2 j}=\frac{s_{j}^{2}}{\hat{\tau}_{j}^{2}} x_{j}^{\operatorname{tr}}\left(\sum_{i=1}^{m} \frac{x_{i}^{\otimes 2}}{\hat{\tau}_{i}}\right)^{-1} x_{j}, \quad \hat{g}_{3 j}=\frac{2 s_{j}^{2}}{\hat{\tau}_{j}^{3}} / \sum_{i=1}^{m} \frac{1}{\hat{\tau}_{i}^{2}}
$$

and it is easy to see that, for large $m$, both $\hat{g}_{2 j}=g_{2 j}+o_{P}\left(m^{-1}\right)$ and $\hat{g}_{3 j}=g_{3 j}+o_{P}\left(m^{-1}\right)$. However, $\hat{g}_{1 j}-g_{1 j}$ has a bias of $\mathcal{O}_{P}\left(m^{-1}\right)$, since according to lemma 1 (formula (9))

$$
\begin{aligned}
E\left(\hat{g}_{1 j}-g_{1 j}\right) & =E\left\{\frac{s_{j}^{2}}{\tau_{j} \hat{\tau}_{j}}\left(\hat{\sigma}^{2}-\sigma^{2}\right)\right\} \\
& =\frac{s_{j}^{2}}{\tau_{j}^{2}} E\left\{\hat{\sigma}^{2}-\sigma^{2}-\frac{\left(\hat{\sigma}^{2}-\sigma^{2}\right)^{2}}{\tau_{j}}\right\}+o\left(m^{-1}\right)
\end{aligned}
$$

which by corollary 1 is equal to

$$
-\Sigma_{\hat{\sigma}^{2}} \frac{s_{j}^{2}}{\tau_{j}^{2}}\left(\sum_{i=1}^{m} \frac{x_{i}^{\mathrm{tr}} \Sigma_{\hat{\beta}} x_{i}}{2 \tau_{i}^{2}}+\frac{1}{\tau_{j}}\right)+o\left(\frac{1}{m}\right)=-\Sigma_{\hat{\sigma}^{2}} \frac{s_{j}^{2}}{\tau_{j}^{2}} \sum_{i=1}^{m} \frac{x_{i}^{\operatorname{tr}} \Sigma_{\hat{\beta}} x_{i}}{2 \tau_{i}^{2}}-g_{3 j}+o\left(\frac{1}{m}\right) .
$$

The result of this development is a second-order-corrected estimator in the untransformed case, which agrees with the Datta and Lahiri (2001) estimator in the Fay-Herriot MLE case with single variance component.

Theorem 4. In the untransformed Fay-Herriot model (1) under assumptions (a)-(c), let

$$
\begin{aligned}
& \hat{\Sigma}_{\hat{\beta}}=\left(\sum_{i=1}^{m} \frac{x_{i}^{\otimes 2}}{\hat{\tau}_{i}}\right)^{-1}, \\
& \hat{\Sigma}_{\hat{\sigma}^{2}}=\left(\sum_{i=1}^{m} \frac{1}{2 \hat{\tau}_{i}^{2}}\right)^{-1} .
\end{aligned}
$$

Then an estimator of $\mathrm{MSE}=E\left(\hat{\vartheta}_{j}-\vartheta_{j}\right)^{2}$ which is unbiased up to remainder $\mathcal{O}\left(m^{-3 / 2}\right)$ is given by

$$
\operatorname{mse}_{j}=\hat{g}_{1 j}+\hat{g}_{2 j}+2 \hat{g}_{3 j}+\frac{s_{j}^{2}}{\hat{\tau}_{j}^{2}} \hat{\Sigma}_{\hat{\sigma}^{2}} \sum_{i=1}^{m} \frac{x_{i}^{\mathrm{tr}} \hat{\Sigma}_{\hat{\beta}} x_{i}}{2 \hat{\tau}_{i}^{2}}
$$

where the estimators $\hat{g}_{k j}$ are as defined above in expression (20).

There is nothing left to prove in this theorem. Again, the newly introduced correction terms are $\mathcal{O}_{P}(1 / m)$, and they clearly estimate and compensate for the corresponding terms of $E\left(\hat{g}_{1 j}-g_{1 j}\right)$ that were calculated above, leaving expected remainders $o_{P}\left(m^{-1}\right)=O_{P}\left(m^{-3 / 2}\right)$.

\subsection{Exponentially transformed case}

Now we proceed to consider the estimation of $\mathrm{MSE}_{j}^{*}$ in the exponentially transformed case. The naïve plug-in estimator is obtained directly by substitution of estimators into the expansion of theorem 3, yielding an expansion in which all other than the first estimate the corresponding terms in the expression of theorem 3 with error $\mathcal{O}_{P}\left(m^{-3 / 2}\right)$. For estimation of the top order term, we again obtain an expression and correct for the bias:

$G_{2 j} \equiv E\left[\exp \left\{2\left(\hat{\eta}_{j}+\hat{\sigma}^{2}\right)\right\}\left\{1-\exp \left(-\hat{\gamma}_{j} s_{j}\right)\right\}-\exp \left\{2\left(\eta_{j}+\sigma^{2}\right)\right\}\left\{1-\exp \left(-\gamma_{j} s_{j}\right)\right\}\right]$ 


$$
\begin{aligned}
= & \exp \left\{2\left(\eta_{j}+\sigma^{2}\right)\right\} E\left(\left[1-\exp \left\{-s_{j}\left(1-\frac{s_{j}}{\tau_{j}}\right)\right\}\right]\left[2 x_{j}^{\operatorname{tr}}(\hat{\beta}-\beta)+2\left\{x_{j}^{\operatorname{tr}}(\hat{\beta}-\beta)\right\}^{2}\right]\right. \\
& +\left[2+\left(\frac{s_{j}^{2}}{\tau_{j}^{2}}-2\right) \exp \left(-\gamma_{j} s_{j}\right)\right]\left(\hat{\sigma}^{2}-\sigma^{2}\right)+\frac{1}{2}\left[2\left\{2+\left(\frac{2 s_{j}^{2}}{\tau_{j}^{2}}-2\right) \exp \left(-\gamma_{j} s_{j}\right)\right\}\right. \\
& \left.\left.-\left(2+\frac{s_{j}^{2}}{\tau_{j}}\right) \frac{s_{j}^{2}}{\tau_{j}^{3}} \exp \left(-\gamma_{j} s_{j}\right)\right]\left(\hat{\sigma}^{2}-\sigma^{2}\right)^{2}+\left\{2+\left(\frac{s_{j}^{2}}{\tau_{j}^{2}}-2\right) \exp \left(-\gamma_{j} s_{j}\right)\right\}\left(\hat{\sigma}^{2}-\sigma^{2}\right) x_{j}^{\operatorname{tr}}(\hat{\beta}-\beta)\right) \\
= & \exp \left\{2\left(\eta_{j}+\sigma^{2}\right)\right\}\left[2\left\{1-\exp \left(-\gamma_{j} s_{j}\right)\right\} x_{j}^{\operatorname{tr}} \Sigma_{\hat{\beta}} x_{j}-\Sigma_{\hat{\sigma}^{2}}\left\{2+\left(\frac{s_{j}^{2}}{\tau_{j}^{2}}-2\right) \exp \left(-\gamma_{j} s_{j}\right)\right\} \sum_{i=1}^{m} \frac{x_{i}^{\operatorname{tr}} \Sigma_{\hat{\beta}} x_{i}}{2 \tau_{i}^{2}}\right. \\
& \left.+\left\{2+\left(\frac{2 s_{j}^{2}}{\tau_{j}^{2}}-2\right) \exp \left(-\gamma_{j} s_{j}\right)-\frac{s_{j}^{2}}{\tau_{j}^{3}} \exp \left(-\gamma_{j} s_{j}\right)\left(1+\frac{s_{j}^{2}}{2 \tau_{j}}\right)\right\} \Sigma_{\hat{\sigma}^{2}}\right]+o\left(\frac{1}{m}\right) .
\end{aligned}
$$

Again it is simple to compensate for this bias by plug-in estimators which are consistent, up to $o\left(\mathrm{~m}^{-1}\right)$ remainders. We record the result by giving a second-order-corrected estimator for the exponentially transformed case.

Theorem 5. Under the Fay-Herriot model (1) and assumptions (a)-(c), where the transformed small area parameter $\vartheta_{j}^{*}=\exp \left(\vartheta_{j}\right)$ is to be estimated by $\hat{\vartheta}_{j}^{*}$ defined in formula (5), an estimator of $\mathrm{MSE}^{*}=E\left(\hat{\vartheta}_{j}^{*}-\vartheta_{j}^{*}\right)^{2}$ which is unbiased up to remainder $\mathcal{O}\left(m^{-3 / 2}\right)$ is given by

$$
\begin{aligned}
\mathrm{mse}_{j}^{*}= & \exp \left\{2\left(\hat{\eta}_{j}+\hat{\sigma}^{2}\right)\right\}\left\{1-\exp \left(-\hat{\gamma}_{j} s_{j}\right)\right\}+\frac{s_{j}^{2}}{\hat{\tau}_{j}^{2}} \exp \left\{2 \hat{\eta}_{j}+\hat{\sigma}^{2}\left(1+\hat{\gamma}_{j}\right)\right\} x_{j}^{\operatorname{tr}} \hat{\Sigma}_{\hat{\beta}} x_{j} \\
& +\hat{\Sigma}_{\hat{\sigma}^{2}} \frac{s_{j}^{2}}{\hat{\tau}_{j}^{2}} \exp \left\{2 \hat{\eta}_{j}+\hat{\sigma}^{2}\left(1+\hat{\gamma}_{j}\right)\right\}\left\{\frac{1}{4}\left(1+3 \hat{\gamma}_{j}\right)^{2}+\frac{1}{\hat{\tau}_{j}}\right\} \\
& -\exp \left\{2\left(\hat{\eta}_{j}+\hat{\sigma}^{2}\right)\right\}\left[2\left\{1-\exp \left(-\hat{\gamma}_{j} s_{j}\right)\right\} x_{j}^{\operatorname{tr}} \hat{\Sigma}_{\beta} x_{j}-\hat{\Sigma}_{\hat{\sigma}^{2}}\left\{2+\left(\frac{s_{j}^{2}}{\hat{\tau}_{j}^{2}}-2\right) \exp \left(-\hat{\gamma}_{j} s_{j}\right)\right\}\right. \\
& \left.\times \sum_{i=1}^{m} \frac{x_{i}^{\operatorname{tr}} \hat{\Sigma}_{\hat{\beta}^{\prime}} x_{i}}{\hat{\tau}_{i}^{2}}+\hat{\Sigma}_{\hat{\sigma}^{2}}\left\{2+\left(\frac{2 s_{j}^{2}}{\hat{\tau}_{j}^{2}}-2\right) \exp \left(-\hat{\gamma}_{j} s_{j}\right)-\frac{s_{j}^{2}}{\hat{\tau}_{j}^{3}} \exp \left(-\hat{\gamma}_{j} s_{j}\right)\left(1+\frac{s_{j}^{2}}{2 \hat{\tau}_{j}}\right)\right\}\right] .
\end{aligned}
$$

\section{Simulation study}

We conducted a simulation study to check the performance of the estimation methodology that was presented in the previous sections. Our simulation design closely imitates the situation that is encountered in the US Census Bureau's on-going 'Small area income and poverty estimation' (SAIPE) project (Citro and Kalton, 2000). For confidentiality, the covariates that we have used are pseudovalues simulated (once only) from a multivariate normal distribution with the same means and variances as the original covariates for all US counties which were used in the SAIPE 1993 log-rate model for poverty among school-age children related to sampled householders. (This was a slight variant of the actual SAIPE production model for income year 1993, which made use of aggregated Current Population Survey (CPS) sampled data for years 1992-1994.) These covariates represent $x_{1}$, the logarithm of the current year Internal Revenue Service estimated child poverty rate for the county, $x_{2}$, the logarithm of the current year county food stamp participation rate, $x_{3}$, the logarithm of the current year county Internal Revenue Service child tax exemptions divided by the current year county population estimate, and $x_{4}$, the logarithm of the county poverty rate for residents aged 5-17 years from the most recent decennial census. We fixed the county covariate values $x_{k i}, 1 \leqslant k \leqslant 4,1 \leqslant i \leqslant 1488$, once and for all. The sample sizes 
Table 1. Data on parameters and estimates in the simulation experiments, based on 10000 replications

\begin{tabular}{|c|c|c|c|c|c|c|c|}
\hline \multirow[t]{2}{*}{$m$} & \multirow[t]{2}{*}{$\bar{n}_{i}$} & \multirow[t]{2}{*}{$\bar{\vartheta}_{i}^{*}$} & \multirow[t]{2}{*}{$s d\left(\vartheta_{i}^{*}\right)$} & \multicolumn{4}{|c|}{$\begin{array}{c}A v\left(\hat{\sigma}^{2}\right) \text { for the following } \\
\text { combinations: }\end{array}$} \\
\hline & & & & $A 1$ & $A 2$ & $B 1$ & $B 2$ \\
\hline 50 & 68 & 0.200 & 0.0847 & 0.065 & 0.066 & 0.374 & 0.404 \\
\hline 100 & 63 & 0.211 & 0.0863 & 0.072 & 0.078 & 0.435 & 0.446 \\
\hline 200 & 78 & 0.191 & 0.0808 & 0.086 & 0.093 & 0.471 & 0.477 \\
\hline 500 & 54 & 0.178 & 0.0833 & 0.092 & 0.094 & 0.485 & 0.488 \\
\hline 1000 & 60 & 0.178 & 0.0875 & 0.096 & 0.097 & 0.493 & 0.494 \\
\hline
\end{tabular}

$n_{i}$ are actual US CPS numbers of sampled households in 1992-1994, as in the SAIPE project, for subsets of the first $m$ alphabetically ordered US counties, with Los Angeles county (which is by far the largest) deleted: this was done because, in simulations which are not reported here, with fixed values of $\sigma^{2}$ as small as 0.014 , we found that the Los Angeles county SAEs were very erratic and distorted the summary measures of the MSE.

We fixed regression coefficients $\beta$ close to the values that were actually fitted in the SAIPE project to the 1993 data (Citro and Kalton, 2000) and generated values $\left\{y_{i}\right\}_{i=1}^{m}$ according to model (1) with $s_{i}=v_{e} / n_{i}$. We have explored various combinations of parameter values $\left(m, \sigma^{2}, v_{e}\right)$ but display results only for $m=50,100,200,500,1000$ and four labelled combinations for $\left(\sigma^{2}, v_{e}\right)$ :

$$
\begin{aligned}
& \begin{array}{llll}
\text { A1 } & \text { A2 } & \text { B1 } & \text { B2 }
\end{array} \\
& \left(\sigma^{2}, v_{e}\right)=(0.1,30) \quad(0.1,17) \quad(0.5,30) \quad(0.5,17) .
\end{aligned}
$$

These parameter values have $\sigma^{2}$ somewhat larger than the values that were fixed in the SAIPE log-count and log-rate models described in Citro and Kalton (2000) but are close to the values that are found in jointly maximizing the Fay-Herriot likelihood with respect to $\left(\beta, \sigma^{2}, v_{e}\right)$. See Slud (2004) for a comparison of the fit and SAEs from these different models.

Table 1 shows the average of sample sizes $n_{i}$ and small area parameters $\vartheta_{i}^{*}$ over areas in the simulation, as a function of the number $m$ of areas included. It also shows the way in which the negative bias of the MLEs $\hat{\sigma}^{2}$ varies with $m$ and with the variance parameter combinations $\sigma^{2}=0.1,0.5$ and $v_{e}=30,17$, indexed by A1, A2, B1 and B2 as shown in expression (24).

This simulation study, unlike studies that have been reported previously in the small area literature, has been designed specifically to make sense in the SAIPE context. The results that are presented focus on the performance of the point estimator (4), on the validity of the theoretical MSE formula that is given in theorem 3 and on the comparison between the proposed MSE estimator (23), its theoretical value and its empirical simulated MSE. We also report the performance of two naïve MSE estimators: first, the naïve application of the Prasad and Rao (1990) formula

$$
\operatorname{mse}_{\mathrm{PRN}}^{*}\left(\hat{\vartheta}_{i}^{*}\right)=\exp \left(2 \hat{\vartheta}_{i}\right) \mathrm{mse}_{i}
$$

where mse $_{i}$ is given by equation (21) and, second, the estimator $\hat{G}_{1 i}$ from equation (18) consisting of only the $O_{P}(1)$ terms in equation (23). In particular, with $R=10000$ simulation replications for each parameter combination, and denoting by index $r$ the random quantities that were simulated and derived on the $r$ th replication, we calculated the following measures of estimator performance: 


$$
\begin{gathered}
T_{0}=\frac{1}{R} \sum_{r=1}^{R}\left\{\hat{\vartheta}_{i}^{*}(r)-\vartheta_{i}^{*}(r)\right\}, \\
T_{1}=E\left\{\operatorname{mse}_{\mathrm{PRN}}^{*}\left(\hat{\vartheta}_{i}^{*}\right)\right\}-\operatorname{SMSE}\left(\hat{\vartheta}_{i}^{*}\right), \\
T_{2}=E\left(\hat{G}_{1 i}\right)-\operatorname{SMSE}\left(\hat{\vartheta}_{i}^{*}\right), \\
T_{3}=E\left(\operatorname{mse}_{i}^{*}\right)-\operatorname{SMSE}\left(\hat{\vartheta}_{i}^{*}\right), \\
T_{4}=\operatorname{SMSE}\left(\hat{\vartheta}_{i}^{*}\right)-\operatorname{MSE}_{i}^{*}, \\
T_{5}=E\left(\operatorname{mse}_{i}^{*}\right)-\operatorname{MSE}_{i}^{*}
\end{gathered}
$$

where $\mathrm{MSE}_{i}^{*}$ and $\mathrm{mse}_{i}^{*}$ are given in theorem 3 and equation (23),

$$
\operatorname{SMSE}\left(\hat{\vartheta}_{i}^{*}\right)=\frac{1}{R} \sum_{r=1}^{R}\left\{\hat{\vartheta}_{i}^{*}(r)-\vartheta_{i}^{*}(r)\right\}^{2}
$$

and the $E(\cdot)$ notation in the equations for $T_{1}, T_{2}, T_{3}$ and $T_{5}$ denotes averaging over simulation replications. Of these summary measures, $T_{0}$ is the only one summarizing SAE bias directly. The empirical MSE estimator SMSE is displayed in Table 2 for comparison with the sizes of absolute MSE errors. The columns $T_{k}$ address the bias of MSE estimation: $T_{1}$ and $T_{2}$ regarding naïve estimators and $T_{3}$ our proposed estimator (23), with $T_{4}$ assessing the theoretical MSE

\begin{tabular}{|c|c|c|c|c|c|c|c|c|c|}
\hline$m$ & Parameters & $T_{0}$ & SMSE & $T_{1}$ & $T_{2}$ & $T_{3}$ & $T_{4}$ & $T_{5}$ & $s d\left(T_{3} / S M S E\right)$ \\
\hline \multirow[t]{4}{*}{50} & A1 & 4.10 & 7.86 & 1.35 & -4.37 & 0.95 & 0.29 & 1.24 & 166.3 \\
\hline & A2 & 1.37 & 5.93 & 0.17 & -2.97 & 0.08 & 0.13 & 0.20 & 109.7 \\
\hline & B1 & 1.76 & 38.44 & -1.14 & -7.72 & -1.96 & 1.62 & -0.34 & 84.6 \\
\hline & $\mathrm{B} 2$ & 0.81 & 28.85 & -1.52 & -4.44 & -0.63 & 0.73 & 0.10 & 75.5 \\
\hline \multirow[t]{4}{*}{100} & A 1 & 1.15 & 6.66 & 0.20 & -2.88 & 0.11 & 0.14 & 0.26 & 73.5 \\
\hline & A2 & 0.38 & 5.54 & -0.12 & -1.88 & -0.09 & 0.08 & -0.01 & 41.1 \\
\hline & B1 & 0.57 & 38.66 & -2.98 & -3.87 & -0.19 & 0.40 & 0.20 & 59.3 \\
\hline & $\mathrm{B} 2$ & 0.14 & 30.73 & -2.94 & -2.60 & -0.14 & 0.00 & -0.14 & 45.5 \\
\hline \multirow[t]{4}{*}{200} & A1 & 0.51 & 4.61 & -0.08 & -1.09 & -0.03 & 0.06 & 0.03 & 22.6 \\
\hline & A2 & 0.15 & 4.00 & -0.10 & -0.67 & -0.02 & 0.02 & -0.00 & 18.7 \\
\hline & B1 & 0.79 & 28.98 & -3.20 & -1.11 & 0.09 & 0.03 & 0.12 & 49.9 \\
\hline & $\mathrm{B} 2$ & 0.42 & 23.78 & -2.69 & -0.08 & 0.04 & 0.01 & 0.04 & 46.4 \\
\hline \multirow[t]{4}{*}{500} & $\mathrm{~A} 1$ & 0.20 & 4.01 & -0.10 & -0.57 & -0.01 & 0.01 & 0.01 & 18.4 \\
\hline & A 2 & -0.02 & 3.64 & -0.13 & -0.37 & -0.02 & 0.00 & -0.02 & 18.5 \\
\hline & B1 & 0.40 & 27.96 & -4.03 & -0.52 & 0.02 & 0.00 & 0.02 & 46.6 \\
\hline & B2 & 0.20 & 23.92 & -3.31 & -0.40 & -0.02 & 0.02 & -0.01 & 45.4 \\
\hline \multirow[t]{4}{*}{1000} & $\mathrm{~A} 1$ & 0.16 & 3.80 & -0.12 & -0.25 & 0.01 & 0.00 & 0.01 & 19.0 \\
\hline & A 2 & 0.05 & 3.50 & -0.12 & -0.16 & 0.00 & 0.00 & 0.00 & 19.3 \\
\hline & B1 & 0.13 & 26.89 & -4.10 & -0.12 & 0.06 & -0.07 & -0.01 & 43.0 \\
\hline & B2 & 0.02 & 22.81 & -3.21 & -0.17 & 0.00 & -0.09 & -0.09 & 43.2 \\
\hline
\end{tabular}
formula (19) and $T_{5}$ showing the degree to which the estimator (23) tracks its theoretical counterpart (19).

Table 2 displays the values of these measures $T_{k}$ averaged over the small areas $i=1, \ldots, m$. In Table 2, we can see in the $T_{0}$-column that the area-averaged biases of the SAEs are small and decrease systematically with $m$. The $T_{1}$-column shows that the naïve estimator mse $\mathrm{PRN}^{*}$ is not

Table 2. Simulation results for MSE estimation $\dagger$

†Values $T_{k}$, and SMSE averaged over areas. Parameter combinations $\left(\sigma^{2}, v_{e}\right)$ are labelled A1-B2 in the second column according to scheme (24). All entries are multiplied by $10^{3}$. 
at all accurate, with MSEs not decreasing as $m$ increases. Next, $T_{2}$ measures the accuracy of the $O(1)$ estimating terms to the full $\mathrm{MSE}_{i}^{*}$, since $\operatorname{SMSE}\left(\hat{\vartheta}_{i}^{*}\right)$ estimates $\operatorname{MSE}_{i}^{*}$ up to $\mathcal{O}\left(m^{-3 / 2}\right)$ according to theorem 3 . Accordingly, we would expect the magnitudes of the $T_{2}$-terms to be of order $1 / m$ as $m$ becomes large, and this is convincingly borne out in the $T_{2}$-column of Table 2 . The measure $T_{3}$ directly compares formula (23) with $\operatorname{SMSE}\left(\hat{\vartheta}_{i}^{*}\right)$. This difference should, according to our theoretical development, decay faster than $1 / m$, and it does so in the $T_{3}$-column of Table 2. (The magnitudes of the final, $m=500$ and $m=1000$, terms are too small to be distinguished effectively by the simulation.) The difference between $T_{2}$ and $T_{3}$ shows that second-order correction in MSE estimation is useful. The comments about $T_{3}$ hold equally well for $T_{4}$ which is the difference between $\operatorname{SMSE}\left(\hat{\vartheta}_{i}^{*}\right)$ and $\mathrm{MSE}_{i}^{*}$ as given by theorem 3 . That theorem says that the differences are $O\left(\mathrm{~m}^{-3 / 2}\right)$, a prediction which is corroborated by the tabulated $T_{4}$-results. Since $T_{5}$ is by definition the sum of $T_{3}$ and $T_{4}$, its behaviour could have been deduced from the behaviour of the $T_{3}$ - and $T_{4}$-columns. The final column of Table 2 is the standard deviation across areas of the ratios $T_{3} / \operatorname{SMSE}\left(\hat{\vartheta}_{i}^{*}\right)$. For example, in the A1 case of $m=100$, the average absolute error $T_{3}$ divided by the average SMSE is $0.112 / 6.66=0.017$, whereas the standard deviation of $T_{3} / \mathrm{SMSE}$ is 0.073 .

The SAIPE and other surveys reporting small area results in transformed models often report variability of small area point estimators through confidence intervals that are formed by backtransforming the confidence intervals formed by using the Prasad-Rao MSE estimator (21). In the setting of our simulation, this would be $\exp \left\{\hat{\vartheta}_{i} \pm z_{\alpha / 2} \sqrt{ } \operatorname{mse}\left(\hat{\vartheta}_{i}\right)\right\}$, where $z_{\alpha / 2}=\Phi^{-1}(1-\alpha / 2)$ is the $(1-\alpha / 2)$-quantile of the standard normal distribution. The half-width of this transformed interval divided by $z_{\alpha / 2}$ provides an alternative naïve MSE estimator and can in fact be Taylor series approximated by $\operatorname{mse}_{\mathrm{PRN}}^{*}\left(\hat{\vartheta}_{i}^{*}\right)$, with very good accuracy when the MSE is small but with poor accuracy otherwise. But the column $T_{1}$ versus $T_{4}$ comparison shows that that approach is not as good as the method that is developed in equation (23).

We have also checked our results against a simulation design that was similar to that of Lahiri and Rao (1995). In particular we have done a small simulation with the choice of $\left(\sigma^{2}, s_{i}\right)$ following Lahiri and Rao's pattern (a). Unlike their constant mean model, we have taken $\eta_{i}=$ $\beta_{0}+\beta_{1} \log \left(x_{i}\right)$, with $x_{i}$ distributed uniform $(0,1), \beta_{0}=0$ and $\beta_{1}=1$. The biases of our mse* (analogous to $T_{3}$ ) are respectively 0.086 and 0.075 for $m=20$ and $m=30$.

\section{Real data example}

We illustrate how the MSE estimates of this paper can be used in the county level SAIPE setting of the previous section, with data for the year 2000. The theme of the example is that (square roots of) MSEs for SAEs provide a handy quantification of the magnitudes of differences between estimates from a model and parameter values from an external source. In other examples, comparisons might similarly be made via the MSE between estimated SAEs and estimates from a competing model.

In the SAIPE 2000 county level data, the response and predictor variables are the same as described in the previous section for 1994, except that the response variables $y_{i}$ are the logtransformed weighted child poverty rates derived from the three years 1999-2001 of CPS data, and the fourth predictor variable $x_{4}$ is the logarithm of the 1990 child poverty rate from the 1990 census (adjusted to reflect the CPS sampling universe and definitions). Data are used only for the 894 counties in both 1990 and 2000 for which the observed (3-year) CPS count of poor related children aged 5-17 years were non-zero. We fitted the Fay-Herriot model (1) to these data, fixing the value $\sigma^{2}=0.016$ as though known and estimating coefficients $\hat{\beta}$ and $\hat{v}_{e}$ by maximum likelihood. The value $\sigma^{2}$ was fixed as in actual SAIPE practice (Citro and Kalton, 2000) at 
Table 3. Descriptive statistics for $\hat{\vartheta}_{i}^{*}, \vartheta_{i}^{\text {cens }}, \mathrm{mse}_{i}^{* 1 / 2}$ and $w_{i}=\left(\hat{\vartheta}_{i}^{*}-\vartheta_{i}^{\text {cens }}\right) / \mathrm{mse}_{i}^{* 1 / 2}$ calculated from SAIPE 2000 data, $i=1, \ldots, 894 \dagger$

\begin{tabular}{|lrrrrrrr|}
\hline Statistic & Minimum & Q1 & Median & Mean & Q3 & Maximum & \multirow{2}{*}{$S D$} \\
\hline & -7.946 & -0.833 & -0.142 & -0.122 & 0.586 & 4.084 & 1.152 \\
$w_{i}$ & 0.021 & 0.104 & 0.143 & 0.154 & 0.191 & 0.507 & 0.073 \\
$\hat{\vartheta}_{i}^{*}$ & 0.018 & 0.100 & 0.142 & 0.158 & 0.200 & 0.589 & 0.084 \\
$\vartheta_{i}^{\text {cens }}$ & 0.004 & 0.014 & 0.018 & 0.020 & 0.025 & 0.074 & 0.010 \\
${\sqrt{ } \mathrm{mse}_{i}^{* 1 / 2}}$ & & & & & & & \\
\hline
\end{tabular}

$\dagger \mathrm{Q} 1$ and Q3 denote quartiles and SD the standard deviation.

the residual variance that was found from a similar log-poverty-rate model fitted to census data; the values throughout the years 1989-2000 ranged between 0.013 and 0.017. With $\sigma^{2}$ fixed and $v_{e}$ estimated, the SAE formulae are exactly as given in equation (5), but the MSEs MSE* and estimators mse $_{i}^{*}$ have modified formulae, given in Slud and Maiti (2005). From these estimates, we constructed the county SAEs (3) for county log-child-poverty rates $\vartheta_{i}$, and SAEs $\hat{\vartheta}_{i}^{*}$. The fit of these SAEs to the target CPS estimands can be assessed in part by comparing them with the corresponding 2000 census child poverty rates adjusted to CPS universe and definitions. (See Kalton and Citro (2000), appendix C, for a similar 1990 comparison; Maiti (2004) considered only a subset of the 1990 SAIPE data; Slud (2003) studied the high correlation between census rates and SAEs in 1990 and 2000.)

How much different are the 2000 SAEs from the census rates? We can view the difference $\hat{\vartheta}_{i}^{*}-\vartheta_{i}^{\text {cens }}=\left(\hat{\vartheta}_{i}^{*}-\vartheta_{i}^{*}\right)+\left(\vartheta_{i}^{*}-\vartheta_{i}^{\text {cens }}\right)$ as the sum of a first term with variance approximately $\mathrm{MSE}_{i}^{*}$ plus a systematic term $\vartheta_{i}^{*}-\vartheta_{i}^{\text {cens }}$ that we have no way of observing separately. However, since the first term divided by $\operatorname{MSE}_{i}^{* 1 / 2}$ should theoretically be approximately a standard normal deviate $z_{i}$, the magnitude of

$$
w_{i} \equiv\left(\hat{\vartheta}_{i}^{*}-\vartheta_{i}^{\text {cens }}\right) / \mathrm{mse}_{i}^{* 1 / 2}
$$

gives a useful measure of the discrepancy between the census and CPS target rates. We summarize the results in Table 3.

For purposes of comparison with the second-order-corrected estimators $\mathrm{mse}_{i}^{*}$ of $\mathrm{MSE}_{i}^{*}$ in this example, we calculated the top order terms $\hat{G}_{1 i}$ as in equation (18). Just as we found in the simulation (the comparison of columns $T_{2}$ and $T_{3}$ in Table 2), the top-order terms are systematically smaller than the second-order-corrected estimators $\mathrm{mse}_{i}^{*}$. Indeed, the deciles (over the set of 894 counties) of the relative differences $\left(\mathrm{mse}_{i}^{*}-\hat{G}_{1 i}\right) / \mathrm{mse}_{i}^{*}$ based on the 2000 SAIPE data were

$\begin{array}{ccccccccc}10 \% & 20 \% & 30 \% & 40 \% & 50 \% & 60 \% & 70 \% & 80 \% & 90 \% \\ 0.041 & 0.054 & 0.063 & 0.074 & 0.084 & 0.096 & 0.121 & 0.155 & 0.220 .\end{array}$

Thus the corrected estimators typically range from $6 \%$ to $16 \%$ higher than the top order, uncorrected, estimators, and confidence intervals based on the corrected versus the uncorrected estimators would typically be $3-8 \%$ wider. This comparison is similar to that in Table 2 , although the setting is different because the variance $\sigma^{2}$ is much smaller in the SAIPE project than in the simulation, and because the SAIPE analysis treats $\sigma^{2}$ and not $v_{e}$ as known.

For most counties, the SAEs and census rates do not differ more than we might expect if the census rates $\vartheta_{i}^{\text {cens }}$ and the true small area CPS target rates $\vartheta_{i}^{*}$ were exactly the same. Thus, the central portion of the histogram (which is not shown) of Studentized deviates $w_{i}$ is roughly symmetric and bell shaped and only slightly less concentrated than a standard normal density. 


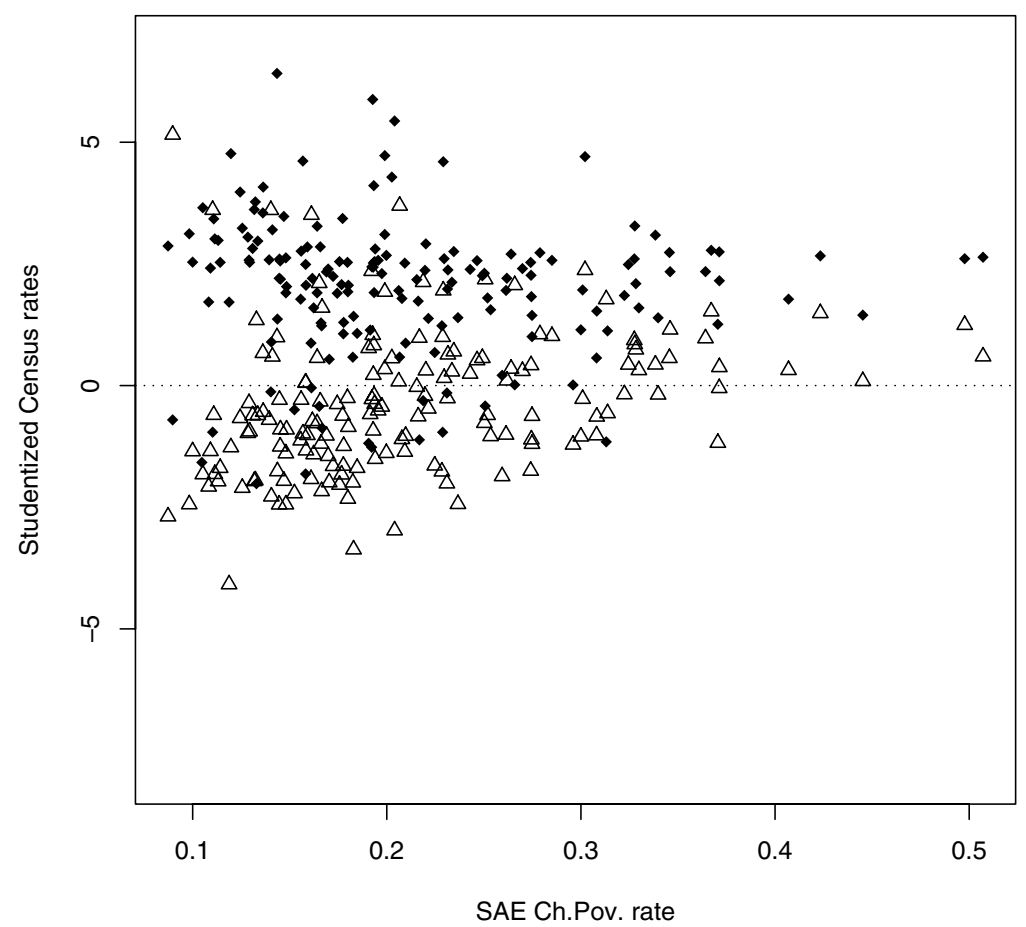

Fig. 1. Scatterplot showing county child poverty rates from the $1990(\diamond)$ and $2000(\triangle)$ censuses, Studentized in the sense that the difference from SAE (5) is divided by MSE ${ }^{1 / 2}$, for the 165 counties where the two census rates differ by 0.05 or more: $\cdots \cdots, y$-co-ordinate for the 2000 census rate

However, the tails of the empirical $w_{i}$-distribution are definitely heavier than normal: 60 of the 894 counties (versus an 'expected' number of 40.5) had $\left|w_{i}\right|>2$ and, in a few counties, values $w_{i}$ with magnitudes between 4 and 8 indicate a real discrepancy between the census rates and the (Fay-Herriot-model-fitted) CPS target rates. Large values $w_{i}$ usually occur in counties for which $\vartheta_{i}^{*}$ changed materially between the 1990 and 2000 censuses.

Fig. 1 plots, against the 2000 SAIPE log-rate model SAEs, the Studentized 1990 and 2000 census rates, centred at the SAEs and scaled by the root-mean-squared error estimates $\mathrm{mse}_{i}^{* 1 / 2}$. Points are plotted only for the 165 counties for which the child poverty rate changed by 0.05 or more between the 1990 and 2000 censuses. Fig. 1 shows the association between Studentized values and between-census changes and also quantifies those changes with respect to rootmean-square units, by county. (In other words, the picture allows us to compare visually the magnitudes of between-census changes and model-based SAE standard errors.) Since the SAEs are based on a model using 2000 SAIPE data, they are generally closer to the 2000 than to the 1990 census values, as expected. In summary, the estimates mse $_{i}^{*}$ are useful in this example for flagging when the SAE versus census 2000 differences are larger than would occur by chance under a Fay-Herriot model.

\section{Conclusions}

This paper provides a 'second-order-corrected' estimation theory for MSEs of small area point estimators based on the Fay and Herriot (1979) model applied to non-linearly transformed survey data. Our emphasis has been on the log-transformed case, but other cases are treated in 
Slud and Maiti (2005). As our data example shows, the MSEs are useful in providing a brief summary of SAE discrepancies from an external standard.

The simulation study of Section 5 covers a broad range of data sizes and parametric settings that are similar to those of the US Census Bureau's SAIPE programme, in the case of log-transformed survey data. The study first shows (column $T_{0}$ of Table 2 ) that the natural bias correction for the small area estimators succeeds excellently in removing bias and generally supports the accuracy of the theoretical MSE formula $\mathrm{MSE}_{j}^{*}$ in theorem 3 and the estimator (23) up to $\mathcal{O}(1 / \mathrm{m})$. The differences in accuracy of MSE estimators with and without second-order correction, both in the simulation and the data example, show that such correction could be important in settings like the SAIPE project.

\section{Acknowledgements}

This paper describes research and analysis of its authors and is released to inform interested parties and to encourage discussion. Results and conclusions are the authors' and have not been endorsed by the US Census Bureau. Most of this research was done at the Census Bureau. The second author's research was supported there by an American Statistical AssociationCensus Fellowship, and also by National Science Foundation grant SES-0318184. We are grateful to Bill Bell for useful conversations regarding methodological issues in the SAIPE project and to the referees for suggestions which improved the presentation.

\section{Appendix A: Proofs of miscellaneous results}

In this appendix, we collect the proofs of various results that are stated in the text above. Throughout, we apply model (1) without comment to express

$$
\begin{gathered}
y_{i}-x_{i}^{\operatorname{tr}} \beta=y_{i}-\eta_{i}=u_{i}+e_{i}, \\
y_{i}-x_{i}^{\operatorname{tr}} \hat{\beta}=u_{i}+e_{i}-x_{i}^{\operatorname{tr}}(\hat{\beta}-\beta) .
\end{gathered}
$$

\section{A.1. Proof of lemma 1}

Our approach is to do a Taylor series expansion around $\left(\beta, \sigma^{2}\right)$ of the exact likelihood equations

$$
\begin{aligned}
\hat{\beta}-\beta & =\left(\sum_{i=1}^{m} \frac{x_{i}^{\otimes 2}}{\hat{\sigma}^{2}+s_{i}}\right)^{-1} \sum_{i=1}^{m} \frac{x_{i}\left(u_{i}+e_{i}\right)}{\hat{\sigma}^{2}+s_{i}}, \\
0 & =\sum_{i=1}^{m} \frac{\left(y_{i}-x_{i}^{\mathrm{tr}} \hat{\beta}\right)^{2}-\hat{\sigma}^{2}-s_{i}}{\left(\hat{\sigma}^{2}+s_{i}\right)^{2}} .
\end{aligned}
$$

The first equation of expression (7) follows immediately from equation (25) and parts (a)-(c), and then, by Taylor series expansion of equation (25) up to second-order terms, we find

$$
\Sigma_{\hat{\beta}}^{-1}(\hat{\beta}-\beta)=\sum_{i=1}^{m} \frac{x_{i}}{\tau_{i}}\left(u_{i}+e_{i}\right)\left(1-\frac{\hat{\sigma}^{2}-\sigma^{2}}{\tau_{i}}\right)+\sum_{i=1}^{m} \frac{x_{i}^{\otimes 2}}{\tau_{i}^{2}} \Sigma_{\hat{\beta}} \sum_{i=1}^{m} \frac{x_{i}}{\tau_{i}}\left(u_{i}+e_{i}\right)\left(\hat{\sigma}^{2}-\sigma^{2}\right)+o_{P}(1) .
$$

Equation (8) follows immediately from this equation on substituting the expression of equation (7) for $\hat{\sigma}^{2}-\sigma^{2}$. find

Next, by Taylor series expansion of equation (26) around $\sigma^{2}$ as a function of $\hat{\sigma}^{2}$, up to second order, we

$$
0=\sum_{i=1}^{m} \frac{\left(y_{i}-x_{i}^{\mathrm{tr}} \hat{\beta}\right)^{2}-\tau_{i}}{\tau_{i}^{2}}+\sum_{i=1}^{m} \frac{\tau_{i}-2\left(y_{i}-x_{i}^{\mathrm{tr}} \hat{\beta}\right)^{2}}{\tau_{i}^{3}}\left(\hat{\sigma}^{2}-\sigma^{2}\right)+\frac{1}{2} \sum_{i=1}^{m} \frac{6\left(y_{i}-x_{i}^{\mathrm{tr}} \hat{\beta}\right)^{2}-2 \tau_{i}}{\tau_{i}^{4}}\left(\hat{\sigma}^{2}-\sigma^{2}\right)^{2}+o_{P}(1) .
$$


The second equation of expression (7) follows immediately from this equation along with the laws of large numbers that are implicit in parts (a)-(c), on dividing through by the coefficient of $\hat{\sigma}^{2}-\sigma^{2}$ and checking that the top order terms arise by substituting $\beta$ for $\hat{\beta}$. In particular, for $k=2,3$, as $m$ becomes large

$$
\sum_{i=1}^{m} \frac{k\left(y_{i}-x_{i}^{\mathrm{tr}} \hat{\beta}\right)^{2}-\tau_{i}}{\tau_{i}^{k+1}}=\sum_{i=1}^{m} \frac{k-1}{\tau_{i}^{k}}+o_{P}(m) .
$$

Next, again dividing through by the coefficient of $\hat{\sigma}^{2}-\sigma^{2}$ and now recognizing that both $\hat{\beta}-\beta$ and $\hat{\sigma}^{2}-\sigma^{2}$ are $\mathcal{O}_{P}\left(m^{-1 / 2}\right)$ by expression $(7)$, we find $\hat{\sigma}^{2}-\sigma^{2}$ equal, with remainder $o_{P}\left(m^{-1}\right)$, to

$$
\begin{aligned}
\left(\sum _ { i = 1 } ^ { m } \frac { 2 } { \tau _ { i } ^ { 3 } } \left[\left(u_{i}+e_{i}\right)^{2}-\right.\right. & \left.\left.\frac{\tau_{i}}{2}-2\left(u_{i}+e_{i}\right) x_{i}^{\operatorname{tr}}(\hat{\beta}-\beta)+\left\{x_{i}^{\operatorname{tr}}(\hat{\beta}-\beta)\right\}^{2}\right]\right)^{-1} \\
& \times \sum_{i=1}^{m}\left[\frac{\left(u_{i}+e_{i}\right)^{2}-2\left(u_{i}+e_{i}\right) x_{i}^{\operatorname{tr}}(\hat{\beta}-\beta)+\left\{x_{i}^{\operatorname{tr}}(\hat{\beta}-\beta)\right\}^{2}-\tau_{i}}{\tau_{i}^{2}}+\frac{2\left(\hat{\sigma}^{2}-\sigma^{2}\right)^{2}}{\tau_{i}^{3}}\right] .
\end{aligned}
$$

Since the first, inverted, term in this last equation is equal to

$$
\frac{1}{2} \Sigma_{\hat{\sigma}^{2}}\left\{1+\Sigma_{\hat{\sigma}^{2}} \sum_{i=1}^{m} \frac{\left(u_{i}+e_{i}\right)^{2}-\tau_{i}}{\tau_{i}^{3}}\right\}^{-1}+\mathcal{O}_{P}\left(\frac{1}{m^{2}}\right)
$$

collecting terms up to order $1 / m$ in the last equation gives equation (9).

\section{A.2. Proof of lemma 2}

The random vector $\left(\alpha_{j}, u_{j}+e_{j}\right)$ is bivariate normal, since it is a linear transformation of the independent normally distributed variables $u_{j}$ and $e_{j}$. Therefore, to check conditional independence it suffices to check that the conditional covariance given $x_{i}$ is 0 :

$$
\begin{aligned}
\operatorname{cov}\left(\alpha_{j}, u_{j}+e_{j}\right) & =\gamma_{j} \operatorname{var}\left(e_{j}\right)-\left(1-\gamma_{j}\right) \operatorname{var}\left(u_{j}\right) \\
& =\frac{\sigma^{2} s_{j}-s_{j} \sigma^{2}}{\tau_{j}}=0 .
\end{aligned}
$$

Since $\left(\alpha_{j}, u_{j}+e_{j}\right)$ is by assumption independent of $\left\{\left(u_{i}, e_{i}\right): i \neq j\right\}$, we conclude that $\alpha_{j}$ is actually conditionally independent of $\left\{u_{i}+e_{i}\right\}_{i=1}^{m}$. The remaining assertion - that $\left(\hat{\beta}, \hat{\sigma}^{2}\right)$ depends on $\left\{\left(u_{i}, e_{i}\right)\right\}_{i=1}^{m}$ only through $\left\{u_{i}+e_{i}\right\}_{i=1}^{m}$-follows by inspection of the likelihood equations (25)-(26) and the standard fact that there is for large $m$ a unique root of the likelihood equations within a small (but fixed) ball around $\left(\beta, \sigma^{2}\right)$.

\section{A.3. Proof of theorem 2}

After substitution within equation (3), we have

$$
\hat{\vartheta}_{j}=\eta_{j}+\left(u_{j}+e_{j}\right) \hat{\gamma}_{j}+x_{j}^{\operatorname{tr}}(\hat{\beta}-\beta)\left(1-\hat{\gamma}_{j}\right) .
$$

Now replace the function $1-\hat{\gamma}_{j}$ of $\hat{\sigma}^{2}$ by a Taylor series approximation up to second order, leaving only $o_{P}(1 / m)$ remainders, obtaining

$$
\begin{aligned}
\hat{\vartheta}_{j}= & \eta_{j}+\left(u_{j}+e_{j}\right) \gamma_{j}+x_{j}^{\operatorname{tr}}(\hat{\beta}-\beta)\left(1-\gamma_{j}\right)+\frac{\left\{u_{j}+e_{j}-x_{j}^{\operatorname{tr}}(\hat{\beta}-\beta)\right\} s_{j}}{\tau_{j}^{2}}\left(\hat{\sigma}^{2}-\sigma^{2}\right) \\
& -\frac{\left(u_{j}+e_{j}\right) s_{j}}{\tau_{j}^{3}}\left(\hat{\sigma}^{2}-\sigma^{2}\right)^{2}+o_{P}\left(\frac{1}{m}\right) .
\end{aligned}
$$

Finally, substituting for $\hat{\beta}-\beta$ and $\hat{\sigma}^{2}-\sigma^{2}$ from equations (8)-(9) leads to 


$$
\begin{aligned}
\hat{\vartheta}_{j}= & \eta_{j}+\left(u_{j}+e_{j}\right) \gamma_{j}+\left(1-\gamma_{j}\right) x_{j}^{\mathrm{tr}}\left\{\Sigma_{\hat{\beta}} \sum_{i=1}^{m} \frac{x_{i}}{\tau_{i}}\left(u_{i}+e_{i}\right)-\Sigma_{\hat{\beta}} \sum_{i=1}^{m} \frac{x_{i}}{\tau_{i}^{2}}\left(u_{i}+e_{i}\right) \Sigma_{\hat{\sigma}^{2}} \sum_{i=1}^{m} \frac{\left(u_{i}+e_{i}\right)^{2}-\tau_{i}}{2 \tau_{i}^{2}}\right. \\
& \left.+\Sigma_{\hat{\beta}} \sum_{i=1}^{m} \frac{x_{i}^{\otimes 2}}{\tau_{i}^{2}} \Sigma_{\hat{\beta}} \sum_{i=1}^{m} \frac{x_{i}}{\tau_{i}}\left(u_{i}+e_{i}\right) \Sigma_{\hat{\sigma}^{2}} \sum_{i=1}^{m} \frac{\left(u_{i}+e_{i}\right)^{2}-\tau_{i}}{2 \tau_{i}^{2}}\right\}+\frac{\left(u_{j}+e_{j}\right) s_{j}}{\tau_{j}^{2}}\left(\Sigma_{\hat{\sigma}^{2}}^{1 / 2} Z_{j}^{(2)}+\Sigma_{\hat{\sigma}^{2}} \frac{\left(u_{j}+e_{j}\right)^{2}-\tau_{j}}{2 \tau_{j}^{2}}\right. \\
& +\Sigma_{\hat{\sigma}^{2}} \sum_{i=1}^{m}\left[\frac{-\left(u_{i}+e_{i}\right) x_{i}^{\mathrm{tr}}}{\tau_{i}^{2}}(\hat{\beta}-\beta)+\frac{\left\{x_{i}^{\mathrm{tr}}(\hat{\beta}-\beta)\right\}^{2}}{2 \tau_{i}^{2}}+\frac{\left(\hat{\sigma}^{2}-\sigma^{2}\right)^{2}}{\tau_{i}^{3}}\right] \\
& \left.-\Sigma_{\hat{\sigma}^{2}}^{2} \sum_{i=1}^{m} \frac{\left(u_{i}+e_{i}\right)^{2}-\tau_{i}}{2 \tau_{i}^{2}} \sum_{i=1}^{m} \frac{\left(u_{i}+e_{i}\right)^{2}-\tau_{i}}{\tau_{i}^{3}}\right)-\frac{s_{j} x_{j}^{\mathrm{tr}}}{\tau_{j}^{2}} \Sigma_{\hat{\beta}}^{1 / 2} Z_{j}^{(1)} \Sigma_{\hat{\sigma}^{2}}^{1 / 2} Z_{j}^{(2)}-\frac{\left(u_{j}+e_{j}\right) s_{j}}{\tau_{j}^{3}} \Sigma_{\hat{\sigma}^{2}}\left(Z_{j}^{(2)}\right)^{2}+o_{P}\left(\frac{1}{m}\right) .
\end{aligned}
$$

All the retained terms in this formula are of order $m^{-1}$ or larger. Now arrange the terms in $\hat{\vartheta}-\vartheta$ with highest order first to obtain equation (14). The alteration of sums to run only over $i \neq j$ would change equation (14) by an amount $\mathcal{O}\left(m^{-3 / 2}\right)$.

\section{A.4. Proof of lemma 4}

(a) This result is essentially a corollary of lemma 2 . Note first that $\alpha_{j}=\gamma_{j} e_{j}-\left(1-\gamma_{j}\right) u_{j}$ is normally distributed with mean 0 and variance $E\left(\alpha_{j}^{2}\right)=\gamma_{j}^{2} s_{j}+\left(1-\gamma_{j}\right)^{2} \sigma^{2}=\left(\sigma^{2} s_{j} / \tau_{j}^{2}\right) \tau_{j}=s_{j} \gamma_{j}$, so that $E\left\{\exp \left(-s_{j} \gamma_{j} / 2+\alpha_{j}\right)\right\}=1$. Then we calculate

$$
\begin{aligned}
& E\left\{\left(u_{j}+e_{j}\right)^{k}\left(\exp \left[\gamma_{j}\left\{s_{j}+2\left(u_{j}+e_{j}\right)\right\}\right]-\exp \left\{\gamma_{j}\left(s_{j} / 2+u_{j}+e_{j}\right)+u_{j}\right\}\right)\right\} \\
& =E\left[\left(u_{j}+e_{j}\right)^{k} \exp \left[\gamma_{j}\left\{s_{j}+2\left(u_{j}+e_{j}\right)\right\}\right]\left\{1-\exp \left(-s_{j} \gamma_{j} / 2-\alpha_{j}\right)\right\}\right]=0
\end{aligned}
$$

where the last equality follows from the independence of $\alpha_{j}$ and $u_{j}+e_{j}$.

(b) This result simply repeats equation (16) for $k=0,1,2$, by inserting $a^{2}=\tau_{j}$ and $b=2 \gamma_{j}$, into the formulae $E\{\exp (a b Z)\}=\exp \left\{(a b)^{2} / 2\right\}$ and

$$
E\left\{(a Z)^{k} \exp (a b Z)\right\}=\exp \left\{(a b)^{2} / 2\right\} \times \begin{cases}a^{2} b & \text { for } k=1, \\ a^{4} b^{2}+a^{2} & \text { for } k=2 .\end{cases}
$$

\section{A.5. Proof of theorem 3}

By equations (17) and (3) and the definition of $\alpha_{j}$,

$$
\vartheta_{j}+\alpha_{j}=\eta_{j}+\gamma_{j}\left(u_{j}+e_{j}\right)
$$

and

$$
\operatorname{MSE}_{j}^{*}=E\left(\exp \left(2 \eta_{j}\right)\left[\exp \left\{\left(\hat{\vartheta}_{j}-\vartheta_{j}-\alpha_{j}\right)+\gamma_{j}\left(u_{j}+e_{j}+s_{j} / 2\right)+\left(\hat{\gamma}_{j}-\gamma_{j}\right) s_{j} / 2\right\}-\exp \left(u_{j}\right)\right]^{2}\right) .
$$

Now substitute equation (14) for $\hat{\vartheta}_{j}-\vartheta_{j}-\alpha_{j}$, noting that the term $\left(\hat{\gamma}_{j}-\gamma_{j}\right) s_{j} / 2$ in the exponent within the square bracket has the effect of changing $u_{j}+e_{j}$ to $u_{j}+e_{j}+s_{j} / 2$ within line (27) and within the corresponding terms of formula (14), yielding $\mathrm{MSE}_{j}^{*}$ up to remainder $\mathcal{O}\left(\mathrm{m}^{-3 / 2}\right)$ as

$$
\begin{aligned}
\exp & \left(2 \eta_{j}\right) E\left(\operatorname { e x p } \{ \gamma _ { j } ( u _ { j } + e _ { j } + \frac { s _ { j } } { 2 } ) \} \left[1+\left(1-\gamma_{j}\right) x_{j}^{\mathrm{tr}} \Sigma_{\hat{\beta}}^{1 / 2} Z_{j}^{(1)}+\frac{1-\gamma_{j}}{\tau_{j}}\left(u_{j}+e_{j}\right) x_{j}^{\mathrm{tr}} \Sigma_{\hat{\beta}} x_{j}+\frac{1-\gamma_{j}}{\tau_{j}}\left(u_{j}+e_{j}+\frac{s_{j}}{2}\right)\right.\right. \\
& \times\left[\Sigma_{\hat{\sigma}^{2}}^{1 / 2} Z_{j}^{(2)}+\frac{1}{2 \tau_{j}} \Sigma_{\hat{\sigma}^{2}}\left\{\frac{\left(u_{j}+e_{j}\right)^{2}}{\tau_{j}}-1\right\}\right]+\left(1-\gamma_{j}\right) x_{j}^{\mathrm{tr}} \Sigma_{\hat{\beta}}\left\{-\sum_{i=1}^{m} \frac{x_{i}}{\tau_{i}^{2}}\left(u_{i}+e_{i}\right) \Sigma_{\hat{\sigma}^{2}} \sum_{i=1}^{m} \frac{\left(u_{i}+e_{i}\right)^{2}-\tau_{i}}{2 \tau_{i}^{2}}\right. \\
& \left.+\sum_{i=1}^{m} \frac{x_{i}^{\otimes 2}}{\tau_{i}^{2}} \Sigma_{\hat{\beta}} \sum_{i=1}^{m} \frac{x_{i}}{\tau_{i}}\left(u_{i}+e_{i}\right) \Sigma_{\hat{\sigma}^{2}} \sum_{i=1}^{m} \frac{\left(u_{i}+e_{i}\right)^{2}-\tau_{i}}{2 \tau_{i}^{2}}\right\}+\frac{\left(u_{j}+e_{j}+s_{j} / 2\right) s_{j}}{\tau_{j}^{2}} \Sigma_{\hat{\sigma}^{2}} \sum_{i=1}^{m}\left\{\frac{-\left(u_{i}+e_{i}\right) x_{i}^{\mathrm{tr}}}{\tau_{i}^{2}} \Sigma_{\hat{\beta}}^{1 / 2} Z_{j}^{(1)}\right. \\
& \left.+\frac{x_{i}^{\mathrm{tr}} \Sigma_{\hat{\beta}} x_{i}}{2 \tau_{i}^{2}}\left(Z_{j}^{(1)}\right)^{2}+\frac{\Sigma_{\hat{\sigma}^{2}}\left(Z_{j}^{(2)}\right)^{2}}{\tau_{i}^{3}}\right\}-\frac{\left(u_{j}+e_{j}+s_{j} / 2\right) s_{j}}{\tau_{j}^{2}} \Sigma_{\hat{\sigma}^{2}}^{2} \sum_{i=1}^{m} \frac{\left(u_{i}+e_{i}\right)^{2}-\tau_{i}}{2 \tau_{i}^{2}} \sum_{i=1}^{m} \frac{\left(u_{i}+e_{i}\right)^{2}-\tau_{i}}{\tau_{i}^{3}} \\
& -\frac{s_{j} x_{j}^{\mathrm{tr}}}{\tau_{j}^{2}} \Sigma_{\hat{\beta}}^{1 / 2} Z_{j}^{(1)} \Sigma_{\hat{\sigma}^{2}}^{1 / 2} Z_{j}^{(2)}-\frac{s_{j}\left(u_{j}+e_{j}+s_{j} / 2\right)}{\tau_{j}^{3}} \Sigma_{\hat{\sigma}^{2}}\left(Z_{j}^{(2)}\right)^{2} \\
& \left.\left.+\frac{1}{2}\left\{\left(1-\gamma_{j}\right) x_{j}^{\mathrm{tr}} \Sigma_{\hat{\beta}}^{1 / 2} Z_{j}^{(1)}+\frac{1-\gamma_{j}}{\tau_{j}}\left(u_{j}+e_{j}+\frac{s_{j}}{2}\right) \Sigma_{\hat{\sigma}^{2}}^{1 / 2} Z_{j}^{(2)}\right\}^{2}\right]-\exp \left(u_{j}\right)\right)^{2} .
\end{aligned}
$$

We now simplify the terms in this expectation by grouping first the $\mathcal{O}(1)$ term, then the cross-terms of 
order $\mathcal{O}(1 / \sqrt{ } m)$ (which are immediately seen to have expectation 0$)$, then the squares of the $\mathcal{O}_{P}\left(m^{-1 / 2}\right)$ terms and then the other expectation terms of order $\mathcal{O}(1 / m)$. The result, using equation (16) with $k=0$, is

$$
\begin{aligned}
\exp & \left(-2 \eta_{j}\right) \mathrm{MSE}_{j}^{*}+\mathcal{O}\left(m^{-3 / 2}\right)=\exp \left(2 \sigma^{2}\right)\left\{1-\exp \left(-\gamma_{j} s_{j}\right)\right\}+\left(1-\gamma_{j}\right)^{2} \\
& \times E\left\{\left\{x_{j}^{\mathrm{tr}} \Sigma_{\hat{\beta}} x_{j}+\frac{\left(u_{j}+e_{j}+s_{j} / 2\right)^{2}}{\tau_{j}^{2}} \Sigma_{\hat{\sigma}^{2}}\right\}\left(2 \exp \left[\gamma_{j}\left\{s_{j}+2\left(u_{j}+e_{j}\right)\right\}\right]-\exp \left\{\gamma_{j}\left(\frac{s_{j}}{2}+u_{j}+e_{j}\right)+u_{j}\right\}\right)\right\} \\
& +\frac{s_{j} \Sigma_{\hat{\sigma}^{2}}}{\tau_{j}^{3}} E\left\{\left\{\frac{\left(u_{j}+e_{j}\right)^{2}}{\tau_{j}}-1\right\}\left(u_{j}+e_{j}+\frac{s_{j}}{2}\right)\left(\exp \left[\gamma_{j}\left\{s_{j}+2\left(u_{j}+e_{j}\right)\right\}\right]-\exp \left\{\gamma_{j}\left(\frac{s_{j}}{2}+u_{j}+e_{j}\right)+u_{j}\right\}\right)\right\} \\
& +2 \frac{1-\gamma_{j}}{\tau_{j}} E\left\{\left[\left(u_{j}+e_{j}\right) x_{j}^{\mathrm{tr}} \Sigma_{\hat{\beta}} x_{j}+\left(u_{j}+e_{j}+\frac{s_{j}}{2}\right) \Sigma_{\hat{\sigma}^{2}}\left\{-\frac{1}{\tau_{j}}+\sum_{i=1}^{m}\left(\frac{x_{i}^{\mathrm{tr}} \Sigma_{\hat{\beta}} x_{i}}{2 \tau_{i}^{2}}+\frac{\Sigma_{\hat{\sigma}^{2}}}{\tau_{i}^{3}}\right)\right\}\right]\right. \\
& \left.\times\left(\exp \left[\gamma_{j}\left\{s_{j}+2\left(u_{j}+e_{j}\right)\right\}\right]-\exp \left\{\gamma_{j}\left(\frac{s_{j}}{2}+u_{j}+e_{j}\right)+u_{j}\right\}\right)\right\} .
\end{aligned}
$$

All other cross-terms had expectation either of 0 , by direct application of lemma 4 , or of order $\mathcal{O}\left(m^{-3 / 2}\right)$. Further simplification based on direct application of lemma 4 yields

$$
\begin{aligned}
\exp \left(-2 \eta_{j}\right) \mathrm{MSE}_{j}^{*}= & \exp \left(2 \sigma^{2}\right)\left\{1-\exp \left(-\gamma_{j} s_{j}\right)\right\}+\mathcal{O}\left(\frac{1}{m^{3 / 2}}\right)+\exp \left\{\sigma^{2}\left(1+\gamma_{j}\right)\right\}\left(1-\gamma_{j}\right)^{2} \\
& \times\left\{x_{j}^{\mathrm{tr}} \Sigma_{\hat{\beta}} x_{j}+\frac{\Sigma_{\hat{\sigma}^{2}}}{\tau_{j}^{2}}\left(4 \sigma^{4}+\tau_{j}+2 s_{j} \sigma^{2}+\frac{s_{j}^{2}}{4}\right)\right\} .
\end{aligned}
$$

Finally, we simplify algebraically: up to $\mathcal{O}\left(m^{-3 / 2}\right)$ terms,

$$
\begin{aligned}
\exp \left(-2 \eta_{j}\right) \mathrm{MSE}_{j}^{*}= & \exp \left(2 \sigma^{2}\right)\left\{1-\exp \left(-\gamma_{j} s_{j}\right)\right\}+\frac{s_{j}^{2}}{\tau_{j}^{2}} \exp \left\{\sigma^{2}\left(1+\gamma_{j}\right)\right\} x_{j}^{\mathrm{tr}} \Sigma_{\hat{\beta}} x_{j}+\Sigma_{\hat{\sigma}^{2}} \frac{s_{j}^{2}}{\tau_{j}^{2}} \exp \left\{\sigma^{2}\left(1+\gamma_{j}\right)\right\} \\
& \times\left\{\frac{1}{\tau_{j}}+\frac{1}{4 \tau_{j}^{2}}\left(4 \sigma^{2}+s_{j}\right)^{2}\right\} \\
= & \exp \left\{2\left(\eta_{j}+\sigma^{2}\right)\right\}\left\{1-\exp \left(-\gamma_{j} s_{j}\right)\right\}+\frac{s_{j}^{2}}{\tau_{j}^{2}} \exp \left\{2 \eta_{j}+\sigma^{2}\left(1+\gamma_{j}\right)\right\} x_{j}^{\mathrm{tr}} \Sigma_{\hat{\beta}} x_{j} \\
& +\Sigma_{\hat{\sigma}^{2}} \frac{s_{j}^{2}}{\tau_{j}^{2}} \exp \left\{2 \eta_{j}+\sigma^{2}\left(1+\gamma_{j}\right)\right\}\left\{\frac{1}{4}\left(1+3 \gamma_{j}\right)^{2}+\frac{1}{\tau_{j}}\right\} .
\end{aligned}
$$

\section{References}

Citro, C. and Kalton, G. (eds) (2000) Small-area Estimates of School-age Children in Poverty: Evaluation of Current Methodology. Washington DC: National Academy Press.

Datta, G. and Lahiri, P. (2001) A unified measure of uncertainty of estimated best linear unbiased predictors in small area estimation problems. Statist. Sin., 10, 613-627.

Fay, R. and Herriot, R. (1979) Estimates of income for small places: an application of James-Stein procedures to census data. J. Am. Statist. Ass., 74, 341-353.

Ghosh, M. and Rao, J. N. K. (1994) Small Area Estimation: an appraisal. Statist. Sci., 9, 55-93.

Jiang, J., Lahiri, P. and Wan, S.-M. (2002) A unified jackknife theory for empirical best prediction with Mestimation. Ann. Statist., 30, 1782-1810.

Lahiri, P. and Rao, J. N. K. (1995) Robust estimation of mean squared error of small area estimators. J. Am. Statist. Ass., 90, 758-766.

Maiti, T. (2004) Applying Jackknife method of mean squared prediction error estimation in SAIPE. Statist. Transn, 6, 685-695.

Prasad, N. and Rao, J. N. K. (1990) The estimation of mean-squared errors of small-area estimators. $J . A m$. Statist. Ass., 85, 163-171.

Rao, J. N. K. (2003) Small Area Estimation. New York: Wiley.

Slud, E. (2003) Assessing fit of SAIPE models to Census and CPS county child-poverty rates. In Proc. Federal Committee on Statistical Methodology Research Conf., Arlington, Nov.

Slud, E. (2004) Small area estimation errors in SAIPE using GLM versus FH models. Proc. Surv. Res. Meth. Sect. Am. Statist. Ass.

Slud, E. and Maiti, T. (2005) MSE estimation in transformed Fay-Herriot models, with application to SAIPE. Report. US Bureau of the Census, Washington DC. 